\title{
EXISTENCE OF AXIALLY SYMMETRIC WEAK SOLUTIONS TO STEADY MHD WITH NONHOMOGENEOUS BOUNDARY CONDITIONS*
}

\author{
SHANGKUN WENG ${ }^{\dagger}$
}

\begin{abstract}
We establish the existence of axially symmetric weak solutions to steady incompressible magnetohydrodynamics with nonhomogeneous boundary conditions. The key issue is the Bernoulli's law for the total head pressure $\Phi=\frac{1}{2}\left(|\mathbf{u}|^{2}+|\mathbf{h}|^{2}\right)+p$ to a special class of solutions to the inviscid, non-resistive MHD system, where the magnetic field only contains the swirl component.
\end{abstract}

Keywords. Existence, MHD equations, axially symmetric, Bernoulli's law.

AMS subject classifications. 76D05, 35Q35.

\section{Introduction and main results}

Let $\Omega \subset \mathbb{R}^{3}$ be an axially symmetric domain with $C^{2}$-smooth boundary $\partial \Omega=\bigcup_{j=0}^{N} \Gamma_{j}$ consisting of $N+1$ disjoint components $\Gamma_{j}$, i.e.,

$$
\Omega=\Omega_{0} \backslash\left(\cup_{j=1}^{N} \overline{\Omega_{j}}\right), \quad \overline{\Omega_{j}} \subset \Omega_{0}, j=1, \ldots, N,
$$

where $\Gamma_{j}=\partial \Omega_{j}$. Consider the steady magnetohydrodynamics (MHD) equations in $\Omega$ :

$$
\begin{cases}(\mathbf{u} \cdot \nabla) \mathbf{u}+\nabla p=(\mathbf{h} \cdot \nabla) \mathbf{h}+\Delta \mathbf{u}+\nabla \times \mathbf{f}, & \forall x \in \Omega \\ (\mathbf{u} \cdot \nabla) \mathbf{h}-(\mathbf{h} \cdot \nabla) \mathbf{u}=\Delta \mathbf{h}+\nabla \times \mathbf{g}, & \forall x \in \Omega \\ \operatorname{div} \mathbf{u}=\operatorname{div} \mathbf{h}=0, & \forall x \in \Omega \\ \mathbf{u}=\mathbf{a}, \quad \mathbf{h}=\mathbf{b} & \text { on } \partial \Omega\end{cases}
$$

For the existence of weak solutions to the system (1.2), the following compatibility conditions are necessary:

$$
\begin{aligned}
\sum_{j=0}^{N} \mathcal{F}_{j} & :=\sum_{j=0}^{N} \int_{\Gamma_{j}} \mathbf{a} \cdot \mathbf{n} d s=0, \\
\sum_{j=0}^{N} \mathcal{G}_{j} & :=\sum_{j=0}^{N} \int_{\Gamma_{j}} \mathbf{b} \cdot \mathbf{n} d s=0,
\end{aligned}
$$

where $\mathbf{n}$ is the outward unit vector to the boundary $\partial \Omega$.

If the magnetic field $\mathbf{h}$ is absent, then the system (1.2) is reduced to the famous steady Navier-Stokes equations

$$
\left\{\begin{array}{l}
(\mathbf{u} \cdot \nabla) \mathbf{u}+\nabla p=\Delta \mathbf{u}+\nabla \times \mathbf{f}, \quad \forall x \in \Omega \\
\operatorname{div} \mathbf{u}=0 \\
\mathbf{u}=\mathbf{a} \text { on } \partial \Omega
\end{array}\right.
$$

*Received: November 15, 2015; accepted (in revised form): May 7, 2016. Communicated by Mikhail Feldman.

${ }^{\dagger}$ Pohang Mathematics Institute, Pohang University of Science and Technology. Hyoja-Dong San 31, Nam-Gu, Pohang, Gyungbuk, 790-784, Republic of Korea (skwengmath@gmail.com). 
Leray [21] made fundamental contributions to the existence theory and showed the existence of a weak solution $\mathbf{u} \in W^{1,2}(\Omega)$ to the system (1.5) under the stronger assumptions

$$
\mathcal{F}_{j}=\int_{\Gamma_{j}} \mathbf{a} \cdot \mathbf{n} d S=0, \quad j=0,1, \ldots, N
$$

Leray provided two different methods for the existence results in [21]. The first one reduced the nonhomogeneous case to homogeneous case by using the solenoidal extension of boundary value a into $\Omega$, which was successively completed and clarified in $[6,11,20])$. The second one is based on a clever contradiction argument, which was used in $[1,2,12,25]$. However, the problem of whether the systems (1.5) and (1.3) admit a solution or not has been open for long time and is usually referred to as Leray's problem in the literature. For sufficiently small fluxes $\mathcal{F}_{j}$, one can also obtain the existence of weak solutions $[2,6,7,9,10,12,18,25]$. The existence was also known with certain symmetric restrictions on the domain and the boundary data and the forcing term (see [1,8,14,22-24]). Recently, Korobkov, Pileckas, and Russo have made an important breakthrough in a series of papers $[13,15-17]$ on the existence theory without any restrictions on the fluxes. First, in [13], they obtained the existence for a plane domain $\Omega$ with two connected components of the boundary assuming only the inflow condition on the external component. The new ingredients of analysis in [13] are the weak one-sided maximum principle for the total head pressure $\Phi=\frac{1}{2}|\mathbf{u}|^{2}+p$ obtained by the Bernoulli's law for weak solutions to the Euler equations and a divergence form representation of $\Phi$. The Bernoulli's law is based on the Morse-Sard theorem developed in [3]. The spatial axially symmetric case was investigated in [15], where the existence was established without any restrictions on the fluxes, if all components $\Gamma_{j}$ of $\partial \Omega$ intersect the axis of symmetry.

In [16], Korobkov, Pileckas, and Russo finally established the existence of weak solutions $\mathbf{u} \in H^{1}(\Omega)$ to the steady Navier-Stokes with boundary values $\mathbf{a} \in W^{3 / 2,2}(\partial \Omega)$ and the force $\nabla \times \mathbf{f} \in H^{1}(\Omega)$ in 2-D bounded domain or 3-D axially symmetric domain with $C^{2}$-smooth boundary, assuming only the total fluxes are zero. By the Morse-Sard theorem proved in [3], almost all level sets of the stream function $\psi$ are finite unions of $C^{1}$ curves. Based on the clear understanding of the level sets of $\psi$ and $\Phi$, they can construct appropriate integration domains (bounded by smooth level lines) and estimate the upper bound of the $L^{2}$ of $\nabla \Phi$. On the other hand, the length of each of these level lines is bounded from below, and the coarea formula implies a lower bound for the $L^{1}$ norm of $\nabla \Phi$, from which they can derive a contradiction. In the proof given in [16], the Bernoulli's law for the Euler equations plays an essential role.

In this paper, we adapt their idea in [16] to the steady MHD equations. More precisely, we will establish the existence of axially symmetric weak solutions $\mathbf{u}(\mathbf{x})=$ $u_{r}(r, z) \mathbf{e}_{r}+u_{\theta}(r, z) \mathbf{e}_{\theta}+u_{z}(r, z) \mathbf{e}_{z}$ and $\mathbf{h}(\mathbf{x})=h_{\theta}(r, z) \mathbf{e}_{\theta}$ to the system (1.2) with nonhomogeneous boundary values in axially symmetric domains with $C^{2}$ smooth boundary. We introduce some notations. Let $O_{x_{1}}, O_{x_{2}}, O_{x_{3}}$ be coordinate axes in $\mathbb{R}^{3}$ and $\theta=\arctan \left(x_{2} / x_{1}\right), r=\left(x_{1}^{2}+x_{2}^{2}\right)^{1 / 2}, z=x_{3}$ be cylindrical coordinates. Denote by $v_{\theta}, v_{r}, v_{z}$ the projections of the vector $\mathbf{v}$ on the axes $\theta, r, z$. A function $f$ is said to be axially symmetric if it does not depend on $\theta$. A vector-valued function $\mathbf{h}=\left(h_{r}, h_{\theta}, h_{z}\right)$ is called axially symmetric if $h_{r}, h_{\theta}$, and $h_{z}$ do not depend on $\theta$. A vector-valued function $\mathbf{h}^{\prime}=\left(h_{r}, h_{\theta}, h_{z}\right)$ is called axially symmetric with no swirl if $h_{\theta}=0$ while $h_{r}$ and $h_{z}$ do not depend on $\theta$.

We need to use the following symmetry assumptions: 
(SO) $\Omega \subset \mathbb{R}^{3}$ is a bounded domain with $C^{2}$ boundary and $O_{x_{3}}$ is a symmetry axis of $\Omega$.

(AS) The assumptions (SO) are fulfilled and both the boundary value $\mathbf{a} \in W^{3 / 2,2}(\partial \Omega)$ and $\nabla \times \mathbf{f} \in W^{1,2}(\Omega)$ are axially symmetric.

(ASwR) The assumptions (SO) are fulfilled and both the boundary value $\mathbf{a} \in$ $W^{3 / 2,2}(\partial \Omega)$ and $\nabla \times \mathbf{f} \in W^{1,2}(\Omega)$ are axially symmetric without rotation.

(ASoS) The assumptions (SO) are fulfilled and both the boundary value $\mathbf{b} \in$ $W^{3 / 2,2}(\partial \Omega)$ and $\nabla \times \mathbf{g} \in W^{1,2}(\Omega)$ are axially symmetric with only swirl component.

We will use standard notation for Sobolev spaces: $W^{k, q}(\Omega), W_{0}^{k, q}(\Omega), W^{\alpha, q}(\partial \Omega)$, where $\alpha \in(0,1), k \in \mathbb{N}_{0}, q \in[1, \infty]$. Denote by $H(\Omega)$ the subspace of all solenoidal vector fields from $W_{0}^{1,2}(\Omega)$ equipped with the norm $\|\mathbf{u}\|_{H(\Omega)}=\|\nabla \mathbf{u}\|_{L^{2}(\Omega)}$. Denote by $L_{A S}^{q}(\Omega)$ $\left(L_{A S w R}^{q}(\Omega)\right)$ the space of all axially symmetric vector-valued functions (without rotation) in $L^{q}(\Omega)$. Similarly, define the spaces $L_{A S o S}^{q}(\Omega), H_{A S}(\Omega), H_{A S w R}(\Omega), H_{A S o S}^{q}(\Omega)$, $W_{A S}^{1,2}(\Omega), W_{A S w R}^{1,2}(\Omega), W_{A S o S}^{1,2}(\Omega), W_{A S}^{3 / 2,2}(\partial \Omega), W_{A S w R}^{3 / 2,2}(\partial \Omega), W_{A S o S}^{3 / 2,2}(\partial \Omega)$, etc. We denote by $\mathcal{H}^{1}$ the one-dimensional Hausdorff measure, i.e., $\mathcal{H}^{1}(F)=\lim _{t \rightarrow 0+} \mathcal{H}_{t}^{1}(F)$, where

$$
\mathcal{H}_{t}^{1}(F)=\inf \left\{\sum_{i=1}^{\infty} \operatorname{diam} F_{i}: \operatorname{diam} F_{i} \leq t, F \subset \bigcup_{i=1}^{\infty} F_{i}\right\} .
$$

The main result of this paper is stated as follows.

THEOREM 1.1. Assume that $\Omega \subset \mathbb{R}^{3}$ is a bounded axially symmetric domain of type (1.1) with $C^{2}$-smooth boundary $\partial \Omega$. If $(\nabla \times \mathbf{f}, \nabla \times \mathbf{g}) \in H_{A S}(\Omega) \times H_{A S o S}(\Omega)$, $(\mathbf{a}, \mathbf{b}) \in W_{A S}^{3 / 2,2}(\partial \Omega) \times W_{A S O S}^{3 / 2,2}(\partial \Omega)$ and $\mathbf{a}$ satisfy the compatibility condition (1.3). Then the system (1.2) admits at least one weak axially symmetric solution $(\mathbf{u}, \mathbf{h}) \in H_{A S}(\Omega) \times$ $H_{A S o S}(\Omega)$. Moreover, if $\nabla \times \mathbf{f} \in H_{A S w R}(\Omega)$ and $\mathbf{a} \in W_{A S w R}^{3 / 2,2}(\partial \Omega)$ are axially symmetric with no swirl, then the system (1.2) admits at least one weak axially symmetric solution with $(\mathbf{u}, \mathbf{h}) \in H_{A S w R}(\Omega) \times H_{A S o S}(\Omega)$.

REMARK 1.1. In the case that $\mathbf{b}=b_{\theta}(r, z) \mathbf{e}_{\theta}$, Equation (1.4) holds automatically since $\mathbf{e}_{\theta} \cdot \mathbf{n} \equiv 0$ on $\partial \Omega$.

For the stationary MHD equations (1.2), we can define the total head pressure $\Phi=$ $\frac{1}{2}\left(|\mathbf{u}|^{2}+|\mathbf{h}|^{2}\right)+p$. Suppose $(\mathbf{u}, \mathbf{h}, p)$ are a smooth solution to the inviscid, non-resistive MHD system. Then we only have

$$
(\mathbf{u} \cdot \nabla) \Phi=(\mathbf{h} \cdot \nabla)(\mathbf{u} \cdot \mathbf{h}) .
$$

So even in the two-dimensional case, the right side is not zero in general. In particular, the level sets of the stream function $\psi$ and $\Phi$ do not coincide with each other; the Bernoulli's law is lost. However, if we further restrict ourselves to the axially symmetric MHD case with the special solution form $\mathbf{u}(\mathbf{x})=u_{r}(r, z) \mathbf{e}_{r}+u_{\theta}(r, z) \mathbf{e}_{\theta}+u_{z}(r, z) \mathbf{e}_{z}$ and $\mathbf{h}(\mathbf{x})=h_{\theta}(r, z) \mathbf{e}_{\theta}$, then $(\mathbf{h} \cdot \nabla)(\mathbf{u} \cdot \mathbf{h})=\frac{h_{\theta}}{r} \partial_{\theta}(\mathbf{u} \cdot \mathbf{h}) \equiv 0$ and the Bernoulli's law holds

$$
(\mathbf{u} \cdot \nabla) \Phi=0
$$

This has been observed in our previous paper [4], where we have used this to prove some Liouville-type theorems for the steady MHD equations. Here, we will adapt the methods developed in [16] to establish the existence of axially weak solutions to the system (1.2). 
This paper is organized as follows. We first prepare some preliminaries to reduce the existence problem to some uniform estimates needed in Lemma 2.5 and Lemma 2.6. Then, in Section 3.1, we first run the Leray's reductio ad absurdum argument for the steady MHD equations. The Bernoulli's law for the inviscid, nonresistive MHD equations is obtained in Section 3.2. Finally, we adapt the methods developed in [16] to the steady MHD equation to obtain a contradiction.

\section{Preliminaries}

The following lemmas concern the existence of solenoidal extensions of boundary values.

LEMMA 2.1.

(i) If $\mathbf{a} \in W_{A S}^{3 / 2,2}(\partial \Omega)$ and Equation (1.3) holds, then there exists an axially symmetric solenoidal extension $\mathbf{A} \in W^{2,2}(\Omega)$ of $\mathbf{a}$ with the estimate

$$
\|\mathbf{A}\|_{W_{A S}^{2,2}(\Omega)} \leq c\|\mathbf{a}\|_{W_{A S}^{3 / 2,2}(\partial \Omega)} .
$$

Moreover, if conditions ( $\mathbf{A S w R}$ ) are prescribed, then $\mathbf{A}$ can be chosen to have zero swirl component.

(ii) If $\mathbf{b} \in W_{A S o S}^{3 / 2,2}(\partial \Omega)$, then there exists a unique vector field $\mathbf{H} \in W_{A S o S}^{2,2}(\Omega)$ such that

$$
\Delta \mathbf{H}=0 \quad \text { in } \Omega, \quad \operatorname{div} \mathbf{H}=0 \quad \text { in } \Omega, \quad \mathbf{H}=\mathbf{b} \quad \text { on } \partial \Omega .
$$

We also have the estimate

$$
\|\mathbf{H}\|_{W_{A S O S}^{2,2}(\Omega)} \leq c\|\mathbf{b}\|_{W_{A S O S}^{3 / 2,2}(\partial \Omega)} .
$$

Proof. The conclusion (i) has been proved in [15]. (ii) Let $\mathbf{b} \in W_{A S o S}^{3 / 2,2}(\partial \Omega)$. Then there exists a unique vector field $\mathbf{F} \in W^{2,2}(\partial \Omega)$ to the Laplace equation

$$
\Delta \mathbf{F}=0 \quad \text { in } \Omega, \quad \mathbf{F}=\mathbf{b} \quad \text { on } \partial \Omega .
$$

By similar arguments as in Lemma 2.2 in [15], we can choose $\mathbf{F}$ to be axially symmetric. By the standard formulas for $\Delta$ in cylindrical coordinate system, one has for $\mathbf{F}=\left(F_{r}, F_{\theta}, F_{z}\right)$

$$
\Delta \mathbf{F}=\left(\Delta_{2}-\frac{1}{r^{2}}\right) F_{r} \mathbf{e}_{r}+\left(\Delta_{2}-\frac{1}{r^{2}}\right) F_{\theta} \mathbf{e}_{\theta}+\left(\Delta_{2} F_{z}\right) \mathbf{e}_{z}=0,
$$

where $\Delta_{2}=\left(\partial_{r}^{2}+\frac{1}{r} \partial_{r}+\partial_{z}^{2}\right)$. Take $\mathbf{H}=F_{\theta} \mathbf{e}_{\theta}$. Then $\mathbf{H} \in W_{A S o S}^{2,2}(\Omega)$, and it follows easily from Equation (2.5) that

$$
\Delta \mathbf{H}=0 .
$$

Since $\mathbf{b} \in W_{A S o S}^{3 / 2,2}(\partial \Omega)$, we still have $\mathbf{H}=\mathbf{b}$ on $\partial \Omega$; therefore, $\mathbf{H}=\mathbf{F}$ by uniqueness. That is, $F_{r}=F_{z} \equiv 0$, which implies that

$$
\operatorname{div} \mathbf{H}=\operatorname{div} \mathbf{F}=\partial_{r} F_{r}+\frac{1}{r} F_{r}+\partial_{z} F_{z}=0 .
$$

REMARK 2.1. The statement and proof of (ii) were suggested by one of the referees. The author would like to thank them for the important improvement. 
Given a function $\mathbf{F} \in L^{q}(\Omega)$ with $q \geq 6 / 5$, consider the continuous linear functional $H(\Omega) \ni \boldsymbol{\eta} \mapsto \int_{\Omega} \mathbf{F} \cdot \boldsymbol{\eta} d x$. By the Riesz representation theorem, there exists a unique function $\mathbf{G} \in H(\Omega)$ with

$$
\int_{\Omega} \mathbf{F} \cdot \boldsymbol{\eta} d x=\int_{\Omega} \nabla \boldsymbol{\eta} \cdot \nabla \mathbf{G} d x=\langle\mathbf{G}, \boldsymbol{\eta}\rangle_{H(\Omega)} \quad \forall \boldsymbol{\eta} \in H(\Omega) .
$$

Put $\mathbf{G}=T_{0} \mathbf{F}$. Evidently, $T_{0}$ is a continuous linear operator from $L^{q}(\Omega)$ to $H(\Omega)$. The following lemmas are easily verified.

LEMma 2.2. The operator $T_{0}: L^{3 / 2}(\Omega) \rightarrow H(\Omega)$ has the following symmetry properties:

$$
\begin{aligned}
& \forall \mathbf{F} \in L_{A S}^{3 / 2}(\Omega) \quad T_{0} \mathbf{F} \in H_{A S}(\Omega), \\
& \forall \mathbf{F} \in L_{A S w R}^{3 / 2}(\Omega) \quad T_{0} \mathbf{F} \in H_{A S w R}(\Omega), \\
& \forall \mathbf{F} \in L_{A S o S}^{3 / 2}(\Omega) \quad T_{0} \mathbf{F} \in H_{A S o S}(\Omega) .
\end{aligned}
$$

LEMMA 2.3. The following inclusions are valid:

$$
\begin{array}{ll}
\forall \mathbf{u}, \mathbf{v} \in H_{A S}(\Omega) & (\mathbf{u} \cdot \nabla) \mathbf{v} \in L_{A S}^{3 / 2}(\Omega), \\
\forall \mathbf{u}, \mathbf{v} \in H_{A S w R}(\Omega) & (\mathbf{u} \cdot \nabla) \mathbf{v} \in L_{A S w R}^{3 / 2}(\Omega), \\
\forall \mathbf{u} \in H_{A S}(\Omega), \mathbf{v} \in H_{A S o S}(\Omega) & (\mathbf{u} \cdot \nabla) \mathbf{v}-(\mathbf{v} \cdot \nabla) \mathbf{u} \in L_{A S o S}^{3 / 2}(\Omega), \\
\forall \mathbf{u}, \mathbf{v} \in H_{A S o S}(\Omega), & (\mathbf{u} \cdot \nabla) \mathbf{v} \in L_{A S w R}^{3 / 2}(\Omega) .
\end{array}
$$

Suppose $\mathbf{a} \in W^{3 / 2,2}(\partial \Omega)$ and also the conditions (1.3) and (AS) (or (ASwR)) are fulfilled. Then we can find a weak axially symmetric solution $\mathbf{U} \in W^{2,2}(\Omega)$ to the Stokes problem in the sense that $\mathbf{U}-\mathbf{A} \in H(\Omega) \cap W^{2,2}(\Omega)$, and the following formula is satisfied by $\mathbf{U}$ :

$$
\int_{\Omega} \nabla \mathbf{U} \cdot \nabla \boldsymbol{\eta} d x=\int_{\Omega}(\nabla \times \mathbf{f}) \cdot \boldsymbol{\eta} d x, \quad \forall \boldsymbol{\eta} \in H(\Omega) .
$$

Moreover,

$$
\|\mathbf{U}\|_{W^{2,2}(\Omega)} \leq c\left(\|\mathbf{a}\|_{W^{3 / 2,2}(\partial \Omega)}+\|\nabla \times \mathbf{f}\|_{L^{2}(\Omega)}\right) .
$$

Put $\mathbf{w}=\mathbf{u}-\mathbf{U}$ and $\mathbf{k}=\mathbf{h}-\mathbf{H}$. Then the problem (1.2) is equivalent to

$$
\left\{\begin{array}{rlrl}
-\Delta \mathbf{w}+ & (\mathbf{U} \cdot \nabla) \mathbf{w}+(\mathbf{w} \cdot \nabla) \mathbf{w}+(\mathbf{w} \cdot \nabla) \mathbf{U}=-\nabla p-(\mathbf{U} \cdot \nabla) \mathbf{U} & \\
& +(\mathbf{H} \cdot \nabla) \mathbf{k}+(\mathbf{k} \cdot \nabla) \mathbf{k}+(\mathbf{k} \cdot \nabla) \mathbf{H}+(\mathbf{H} \cdot \nabla) \mathbf{H}, & & \text { in } \Omega, \\
-\Delta \mathbf{k}+ & (\mathbf{U} \cdot \nabla) \mathbf{k}+(\mathbf{w} \cdot \nabla) \mathbf{k}+(\mathbf{w} \cdot \nabla) \mathbf{H}-(\mathbf{k} \cdot \nabla) \mathbf{U}-(\mathbf{k} \cdot \nabla) \mathbf{w}-(\mathbf{H} \cdot \nabla) \mathbf{w}=0 & \\
& -(\mathbf{U} \cdot \nabla) \mathbf{H}+(\mathbf{H} \cdot \nabla) \mathbf{U}+\nabla \times \mathbf{g}, & & \text { in } \Omega,{ }^{2.8)} \\
\operatorname{div} \mathbf{w}=\operatorname{div} \mathbf{k}=0 & & \text { in } \Omega, \\
\mathbf{w}=\mathbf{k}=0 & & \text { on } \partial \Omega .
\end{array}\right.
$$

By a weak solution to the problem (1.2), we understand functions $(\mathbf{u}, \mathbf{h})$ such that $\mathbf{w}=\mathbf{u}-\mathbf{U} \in H(\Omega), \mathbf{k}=\mathbf{h}-\mathbf{H} \in H(\Omega)$ and for any $\boldsymbol{\eta} \in H(\Omega), \boldsymbol{\zeta} \in W_{0}^{1,2}(\Omega)$

$$
\langle\mathbf{w}, \boldsymbol{\eta}\rangle_{H(\Omega)}=-\int_{\Omega}(\mathbf{U} \cdot \nabla) \mathbf{U} \cdot \boldsymbol{\eta} d x-\int_{\Omega}(\mathbf{U} \cdot \nabla) \mathbf{w} \cdot \boldsymbol{\eta} d x-\int_{\Omega}(\mathbf{w} \cdot \nabla) \mathbf{w} \cdot \boldsymbol{\eta} d x
$$




$$
\begin{aligned}
& -\int_{\Omega}(\mathbf{w} \cdot \nabla) \mathbf{U} \cdot \boldsymbol{\eta} d x+\int_{\Omega}(\mathbf{H} \cdot \nabla) \mathbf{k} \cdot \boldsymbol{\eta} d x+\int_{\Omega}(\mathbf{k} \cdot \nabla) \mathbf{k} \cdot \boldsymbol{\eta} d x \\
& +\int_{\Omega}(\mathbf{k} \cdot \nabla) \mathbf{H} \cdot \boldsymbol{\eta} d x+\int_{\Omega}(\mathbf{H} \cdot \nabla) \mathbf{H} \cdot \boldsymbol{\eta} d x, \\
\langle\mathbf{k}, \boldsymbol{\zeta}\rangle_{H(\Omega)}=- & \int_{\Omega}(\mathbf{U} \cdot \nabla) \mathbf{H} \cdot \boldsymbol{\zeta} d x-\int_{\Omega}(\mathbf{U} \cdot \nabla) \mathbf{k} \cdot \boldsymbol{\zeta} d x-\int_{\Omega}(\mathbf{w} \cdot \nabla) \mathbf{k} \cdot \boldsymbol{\zeta} d x \\
& -\int_{\Omega}(\mathbf{w} \cdot \nabla) \mathbf{H} \cdot \boldsymbol{\zeta} d x+\int_{\Omega}(\mathbf{k} \cdot \nabla) \mathbf{U} \cdot \boldsymbol{\zeta} d x+\int_{\Omega}(\mathbf{k} \cdot \nabla) \mathbf{w} \cdot \boldsymbol{\zeta} \\
& +\int_{\Omega}(\mathbf{H} \cdot \nabla) \mathbf{w} \cdot \boldsymbol{\zeta} d x+\int_{\Omega}(\mathbf{H} \cdot \nabla) \mathbf{U} \cdot \boldsymbol{\zeta} d x+\int_{\Omega}(\nabla \times \mathbf{g}) \cdot \boldsymbol{\zeta} d x .
\end{aligned}
$$

By the Riesz representation theorem, for any $\left(\begin{array}{c}\mathbf{w} \\ \mathbf{k}\end{array}\right) \in H(\Omega) \times H(\Omega)$ there exists a unique element $\mathbb{T}\left(\begin{array}{l}\mathbf{w} \\ \mathbf{k}\end{array}\right)=\left(T_{1}\left(\begin{array}{l}\mathbf{w} \\ \mathbf{k}\end{array}\right), T_{2}\left(\begin{array}{l}\mathbf{w} \\ \mathbf{k}\end{array}\right)\right)^{T} \in H(\Omega) \times H(\Omega)$ such that the right-hand sides of Equation (2.9) are equivalent to $\left\langle T_{1}\left(\begin{array}{l}\mathbf{w} \\ \mathbf{k}\end{array}\right), \boldsymbol{\eta}\right\rangle_{H(\Omega)}$ and $\left\langle T_{2}\left(\begin{array}{l}\mathbf{w} \\ \mathbf{k}\end{array}\right), \boldsymbol{\zeta}\right\rangle_{H(\Omega)}$ for all $\boldsymbol{\eta} \in H(\Omega), \boldsymbol{\zeta} \in W_{0}^{1,2}(\Omega)$, respectively. Obviously, $\mathbb{T}$ is a nonlinear operator from $H(\Omega) \times H(\Omega)$ to $H(\Omega) \times H(\Omega)$.

Lemma 2.4. The operator $\mathbb{T}: H(\Omega) \times H(\Omega) \rightarrow H(\Omega) \times H(\Omega)$ is compact. Moreover, $\mathbb{T}$ has the following symmetry properties:

$$
\begin{aligned}
& \forall\left(\begin{array}{c}
\mathbf{w} \\
\mathbf{k}
\end{array}\right) \in H_{A S}(\Omega) \times H_{A S o S}(\Omega), \quad T_{1}\left(\begin{array}{c}
\mathbf{w} \\
\mathbf{k}
\end{array}\right) \in H_{A S}(\Omega), \\
& \forall\left(\begin{array}{c}
\mathbf{w} \\
\mathbf{k}
\end{array}\right) \in H_{A S w R}(\Omega) \times H_{A S o S}(\Omega), \quad T_{1}\left(\begin{array}{c}
\mathbf{w} \\
\mathbf{k}
\end{array}\right) \in H_{A S w R}(\Omega), \\
& \forall\left(\begin{array}{c}
\mathbf{w} \\
\mathbf{k}
\end{array}\right) \in H_{A S}(\Omega) \times H_{A S o S}(\Omega), \quad T_{2}\left(\begin{array}{c}
\mathbf{w} \\
\mathbf{k}
\end{array}\right) \in H_{A S o S}(\Omega) .
\end{aligned}
$$

Proof. The compactness can be proved in a standard way as shown in [20], and Equation (2.10) follows from Lemma 2.2 and Lemma 2.3.

Hence, Equation (2.9) is equivalent to the operator equation

$$
\left(\begin{array}{l}
\mathrm{w} \\
\mathrm{k}
\end{array}\right)=\mathbb{T}\left(\begin{array}{l}
\mathrm{w} \\
\mathrm{k}
\end{array}\right)
$$

in the space $H(\Omega) \times H(\Omega)$. Thus, we can apply the Leray-Schauder fixed point theorem to the compact operators $\left.\mathbb{T}\right|_{H_{A S}(\Omega) \times H_{A S o S}(\Omega)}$ and $\left.\mathbb{T}\right|_{H_{A S w R}(\Omega) \times H_{A S o S}(\Omega)}$. Then the following statements hold.

LEMma 2.5. Let conditions (AS) and (ASoS) and Equations (1.3)-(1.4) be fulfilled. Suppose all possible solutions $\left(\begin{array}{l}\mathbf{w} \\ \mathbf{k}\end{array}\right)$ to the equation $\left(\begin{array}{l}\mathbf{w} \\ \mathbf{k}\end{array}\right)=\lambda \mathbb{T}\left(\begin{array}{l}\mathbf{w} \\ \mathbf{k}\end{array}\right)$ with $\lambda \in[0,1]$ are uniformly bounded in $H(\Omega) \times H(\Omega)$. Then problem (1.2) admits at least one weak axially symmetric solution $(\mathbf{u}, \mathbf{h}) \in H_{A S}(\Omega) \times H_{A S o S}(\Omega)$.

LEMMA 2.6. Let conditions (ASwR) and (ASoS) and Equations (1.3)-(1.4) be fulfilled. Suppose all possible solutions $\left(\begin{array}{l}\mathbf{w} \\ \mathbf{k}\end{array}\right)$ to the equation $\left(\begin{array}{l}\mathbf{w} \\ \mathbf{k}\end{array}\right)=\lambda \mathbb{T}\left(\begin{array}{l}\mathbf{w} \\ \mathbf{k}\end{array}\right)$ with 
$\lambda \in[0,1]$ are uniformly bounded in $H(\Omega) \times H(\Omega)$. Then problem (1.2) admits at least one weak axially symmetric solution $(\mathbf{u}, \mathbf{h}) \in H_{A S w R}(\Omega) \times H_{A S o S}(\Omega)$.

\section{Proof of Theorem 1.1}

3.1. The reductio ad absurdum argument by Leray. We apply the reductio ad absurdum argument of Leray [21] to the stationary MHD equations. To prove the existence of a weak solution to the MHD system (1.2), by Lemma 2.5, and Lemma 2.6 it is sufficient to show that the weak solutions $(\mathbf{w}, \mathbf{k})$ satisfying for any $(\boldsymbol{\eta}, \boldsymbol{\zeta}) \in$ $H(\Omega) \times W_{0}^{1,2}(\Omega)$

$$
\begin{aligned}
\langle\mathbf{w}, \boldsymbol{\eta}\rangle_{H(\Omega)}=- & \lambda \int_{\Omega}(\mathbf{U} \cdot \nabla) \mathbf{U} \cdot \boldsymbol{\eta} d x-\lambda \int_{\Omega}(\mathbf{U} \cdot \nabla) \mathbf{w} \cdot \boldsymbol{\eta} d x-\lambda \int_{\Omega}(\mathbf{w} \cdot \nabla) \mathbf{w} \cdot \boldsymbol{\eta} d x \\
& -\lambda \int_{\Omega}(\mathbf{w} \cdot \nabla) \mathbf{U} \cdot \boldsymbol{\eta} d x+\lambda \int_{\Omega}(\mathbf{H} \cdot \nabla) \mathbf{k} \cdot \boldsymbol{\eta} d x+\lambda \int_{\Omega}(\mathbf{k} \cdot \nabla) \mathbf{k} \cdot \boldsymbol{\eta} d x \\
& +\lambda \int_{\Omega}(\mathbf{k} \cdot \nabla) \mathbf{H} \cdot \boldsymbol{\eta} d x+\lambda \int_{\Omega}(\mathbf{H} \cdot \nabla) \mathbf{H} \cdot \boldsymbol{\eta} d x \\
\langle\mathbf{k}, \boldsymbol{\zeta}\rangle_{H(\Omega)}=- & \lambda \int_{\Omega}(\mathbf{U} \cdot \nabla) \mathbf{H} \cdot \boldsymbol{\zeta} d x-\lambda \int_{\Omega}(\mathbf{U} \cdot \nabla) \mathbf{k} \cdot \boldsymbol{\zeta} d x-\lambda \int_{\Omega}(\mathbf{w} \cdot \nabla) \mathbf{k} \cdot \boldsymbol{\zeta} d x \\
& -\lambda \int_{\Omega}(\mathbf{w} \cdot \nabla) \mathbf{H} \cdot \boldsymbol{\zeta} d x+\lambda \int_{\Omega}(\mathbf{k} \cdot \nabla) \mathbf{U} \cdot \boldsymbol{\zeta} d x+\lambda \int_{\Omega}(\mathbf{k} \cdot \nabla) \mathbf{w} \cdot \boldsymbol{\zeta} \\
& +\lambda \int_{\Omega}(\mathbf{H} \cdot \nabla) \mathbf{w} \cdot \boldsymbol{\zeta} d x \lambda+\int_{\Omega}(\mathbf{H} \cdot \nabla) \mathbf{U} \cdot \boldsymbol{\zeta} d x-\lambda \int_{\Omega} \nabla \mathbf{H} \cdot \nabla \boldsymbol{\zeta} d x+\lambda \int_{\Omega}(\nabla \times \mathbf{g}) \cdot \boldsymbol{\zeta} d x
\end{aligned}
$$

are uniformly bounded in $H(\Omega) \times H(\Omega)$ with respect to $\lambda \in[0,1]$. Assume that this is false. Then there exist sequences $\left\{\lambda_{n}\right\}_{n \in \mathbb{N}} \subset[0,1]$ and $\left\{\widehat{\mathbf{w}}_{n}, \widehat{\mathbf{k}}_{n}\right\}_{n \in \mathbb{N}} \in H(\Omega) \times H(\Omega)$ such that, for any $(\boldsymbol{\eta}, \boldsymbol{\zeta}) \in H(\Omega) \times W_{0}^{1,2}(\Omega)$,

$$
\begin{aligned}
& \int_{\Omega} \nabla \widehat{\mathbf{w}}_{n} \cdot \nabla \boldsymbol{\eta} d x-\lambda_{n} \int_{\Omega}\left(\left(\widehat{\mathbf{w}}_{n}+\mathbf{U}\right) \cdot \nabla\right) \boldsymbol{\eta} \cdot \widehat{\mathbf{w}}_{n} d x-\lambda_{n} \int_{\Omega}\left(\widehat{\mathbf{w}}_{n} \cdot \nabla\right) \boldsymbol{\eta} \cdot \mathbf{U} d x \\
& \quad+\lambda_{n} \int_{\Omega}\left(\left(\widehat{\mathbf{k}}_{n}+\mathbf{H}\right) \cdot \nabla\right) \boldsymbol{\eta} \cdot \widehat{\mathbf{k}}_{n} d x+\lambda_{n} \int_{\Omega}\left(\widehat{\mathbf{k}}_{n} \cdot \nabla\right) \boldsymbol{\eta} \cdot \mathbf{H} d x \\
& =\lambda_{n} \int_{\Omega}(\mathbf{U} \cdot \nabla) \boldsymbol{\eta} \cdot \mathbf{U} d x-\lambda_{n} \int_{\Omega}(\mathbf{H} \cdot \nabla) \boldsymbol{\eta} \cdot \mathbf{H} d x, \\
& \quad \int_{\Omega} \nabla \widehat{\mathbf{k}}_{n} \cdot \nabla \boldsymbol{\zeta} d x-\lambda_{n} \int_{\Omega}\left(\left(\widehat{\mathbf{w}}_{n}+\mathbf{U}\right) \cdot \nabla\right) \boldsymbol{\zeta} \cdot \widehat{\mathbf{k}}_{n} d x-\lambda_{n} \int_{\Omega}\left(\widehat{\mathbf{w}}_{n} \cdot \nabla\right) \boldsymbol{\zeta} \cdot \mathbf{H} d x \\
& \quad+\lambda_{n} \int_{\Omega}\left(\left(\widehat{\mathbf{k}}_{n}+\mathbf{H}\right) \cdot \nabla\right) \boldsymbol{\zeta} \cdot \widehat{\mathbf{w}}_{n} d x+\lambda_{n} \int_{\Omega}\left(\widehat{\mathbf{k}}_{n} \cdot \nabla\right) \boldsymbol{\zeta} \cdot \mathbf{U} d x \\
& =\lambda_{n} \int_{\Omega}((\mathbf{U} \cdot \nabla) \boldsymbol{\zeta}) \cdot \mathbf{H} d x-\lambda_{n} \int_{\Omega}(\mathbf{H} \cdot \nabla) \boldsymbol{\zeta} \cdot \mathbf{U} d x-\lambda_{n} \int_{\Omega} \nabla \mathbf{H} \cdot \nabla \boldsymbol{\zeta} d x+\lambda_{n} \int_{\Omega}(\nabla \times \mathbf{g}) \cdot \boldsymbol{\zeta} d x
\end{aligned}
$$

and

$$
\lim _{n \rightarrow \infty} \lambda_{n}=\lambda_{0} \in[0,1], \quad \lim _{n \rightarrow \infty} J_{n}^{2}=\lim _{n \rightarrow \infty}\left(\left\|\widehat{\mathbf{w}}_{n}\right\|_{H(\Omega)}^{2}+\left\|\widehat{\mathbf{k}}_{n}\right\|_{H(\Omega)}^{2}\right)=\infty .
$$

Denote $\mathbf{w}_{n}=J_{n}^{-1} \widehat{\mathbf{w}}_{n}, \mathbf{k}_{n}=J_{n}^{-1} \widehat{\mathbf{k}}_{n}$. Since $\left\|\mathbf{w}_{n}\right\|_{H(\Omega)}^{2}+\left\|\mathbf{k}_{n}\right\|_{H(\Omega)}^{2}=1$, there exists a subsequence $\left\{\mathbf{w}_{n_{l}}, \mathbf{k}_{n_{l}}\right\}$ converging weakly in $H(\Omega)$ to vector fields $\mathbf{w}, \mathbf{k} \in H(\Omega)$. Because 
of the compact embedding

$$
H(\Omega) \mapsto L^{r}(\Omega) \quad \forall r \in[1,6),
$$

the subsequence $\left\{\mathbf{w}_{n_{l}}, \mathbf{k}_{n_{l}}\right\}$ converges strongly in $L^{r}(\Omega)$. Replacing $\zeta$ in Equation (3.3) by $J_{n}^{-2} \zeta$ and letting $n \rightarrow \infty$, we obtain

$$
\lambda_{0} \int_{\Omega}[(\mathbf{w} \cdot \nabla) \mathbf{k}-(\mathbf{k} \cdot \nabla) \mathbf{w}] \cdot \zeta d x=0 .
$$

Taking $\boldsymbol{\eta}=J_{n}^{-2} \widehat{\mathbf{w}}_{n}, \boldsymbol{\zeta}=J_{n}^{-2} \widehat{\mathbf{k}}_{n}$ in Equations (3.2)-(3.3) and adding the above two identities, we get

$$
\begin{aligned}
& \int_{\Omega}\left|\nabla \mathbf{w}_{n}\right|^{2}+\left|\nabla \mathbf{k}_{n}\right|^{2} d x \\
&=\lambda_{n} \int_{\Omega}\left[\left(\mathbf{w}_{n} \cdot \nabla\right) \mathbf{w}_{n}-\left(\mathbf{k}_{n} \cdot\right) \mathbf{k}_{n}\right] \cdot \mathbf{U} d x-\lambda_{n} \int_{\Omega}\left[\left(\mathbf{w}_{n} \cdot \nabla\right) \mathbf{k}_{n}-\left(\mathbf{k}_{n} \cdot \nabla\right) \mathbf{w}_{n}\right] \cdot \mathbf{H} d x \\
& \quad+J_{n}^{-1} \lambda_{n} \int_{\Omega}\left[(\mathbf{U} \cdot \nabla) \mathbf{w}_{n} \cdot \mathbf{U}-(\mathbf{H} \cdot \nabla) \mathbf{w}_{n} \cdot \mathbf{H}+(\mathbf{U} \cdot \nabla) \mathbf{k}_{n} \cdot \mathbf{H}-(\mathbf{H} \cdot \nabla) \mathbf{k}_{n} \cdot \mathbf{U}\right] d x \\
& \quad-J_{n}^{-1} \lambda_{n} \int_{\Omega}\left[(\nabla \times \mathbf{g}) \cdot \mathbf{k}_{n}+\nabla \mathbf{H} \cdot \nabla \mathbf{k}_{n}\right] d x .
\end{aligned}
$$

Therefore, passing to a limit as $n_{l} \rightarrow \infty$ in Equation (3.6) and using Equation (3.5), we obtain

$$
1=\lambda_{0} \int_{\Omega}[(\mathbf{w} \cdot \nabla) \mathbf{w}-(\mathbf{k} \cdot \nabla) \mathbf{k}] \cdot \mathbf{U} d x
$$

This implies $\lambda_{0} \in(0,1]$. Let us return to the integral identity (3.2). Consider the functional

$$
\begin{aligned}
R_{n}(\boldsymbol{\eta})= & \int_{\Omega} \nabla \widehat{\mathbf{w}}_{n} \cdot \nabla \boldsymbol{\eta} d x-\lambda_{n} \int_{\Omega}\left(\left(\widehat{\mathbf{w}}_{n}+\mathbf{U}\right) \cdot \nabla\right) \boldsymbol{\eta} \cdot \widehat{\mathbf{w}}_{n} d x-\lambda_{n} \int_{\Omega}\left(\widehat{\mathbf{w}}_{n} \cdot \nabla\right) \boldsymbol{\eta} \cdot \mathbf{U} d x \\
& +\lambda_{n} \int_{\Omega}\left(\left(\widehat{\mathbf{k}}_{n}+\mathbf{H}\right) \cdot \nabla\right) \boldsymbol{\eta} \cdot \widehat{\mathbf{k}}_{n} d x+\lambda_{n} \int_{\Omega}\left(\widehat{\mathbf{k}}_{n} \cdot \nabla\right) \boldsymbol{\eta} \cdot \mathbf{H} d x-\lambda_{n} \int_{\Omega}(\mathbf{U} \cdot \nabla) \boldsymbol{\eta} \cdot \mathbf{U} d x \\
& +\lambda_{n} \int_{\Omega}(\mathbf{H} \cdot \nabla) \boldsymbol{\eta} \cdot \mathbf{H} d x \quad \forall \boldsymbol{\eta} \in W_{0}^{1,2}(\Omega) .
\end{aligned}
$$

Obviously, $R_{k}(\boldsymbol{\eta})$ is a linear functional and

$$
\left|R_{n}(\boldsymbol{\eta})\right| \leq c\left(\left\|\left(\widehat{\mathbf{w}}_{n}, \widehat{\mathbf{k}}_{n}\right)\right\|_{H(\Omega)}+\left\|\left(\widehat{\mathbf{w}}_{n}, \widehat{\mathbf{k}}_{n}\right)\right\|_{H(\Omega)}^{2}+\|(\mathbf{a}, \mathbf{b})\|_{W^{3 / 2,2}(\partial \Omega)}^{2}+\|\mathbf{f}\|_{W_{0}^{1,2}(\Omega)}^{2}\right)\|\boldsymbol{\eta}\|_{H(\Omega)}
$$

with constant $c$ independent of $n$. It follows from Equation (3.2) that

$$
R_{n}(\boldsymbol{\eta})=0 \quad \forall \boldsymbol{\eta} \in H(\Omega)
$$

Therefore, there exists an axially symmetric function $\hat{p}_{n} \in \hat{L}^{2}(\Omega)=\left\{q \in L^{2}(\Omega)\right.$ : $\left.\int_{\Omega} q(x) d x=0\right\}$ such that

$$
R_{n}(\boldsymbol{\eta})=\int_{\Omega} \hat{p}_{n} \operatorname{div} \boldsymbol{\eta} d x \quad \forall \boldsymbol{\eta} \in W_{0}^{1,2}(\Omega)
$$


and

$$
\left\|\hat{p}_{n}\right\|_{L^{2}(\Omega)} \leq c\left(\left\|\left(\widehat{\mathbf{w}}_{n}, \widehat{\mathbf{k}}_{n}\right)\right\|_{H(\Omega)}+\left\|\left(\widehat{\mathbf{w}}_{n}, \widehat{\mathbf{k}}_{n}\right)\right\|_{H(\Omega)}^{2}+\|(\mathbf{a}, \mathbf{b})\|_{W^{3 / 2,2}(\partial \Omega)}^{2}+\|\mathbf{f}\|_{W_{0}^{1,2}(\Omega)}^{2}\right) .
$$

The pair $\left(\widehat{\mathbf{w}}_{n}, \widehat{\mathbf{k}}_{n}, \hat{p}_{n}\right)$ satisfies the integral identity

$$
\begin{aligned}
& \int_{\Omega} \nabla \widehat{\mathbf{w}}_{n} \cdot \nabla \boldsymbol{\eta} d x-\lambda_{n} \int_{\Omega}\left(\left(\widehat{\mathbf{w}}_{n}+\mathbf{U}\right) \cdot \nabla\right) \boldsymbol{\eta} \cdot \widehat{\mathbf{w}}_{n} d x-\lambda_{n} \int_{\Omega}\left(\widehat{\mathbf{w}}_{n} \cdot \nabla\right) \boldsymbol{\eta} \cdot \mathbf{U} d x \\
& +\lambda_{n} \int_{\Omega}\left(\left(\widehat{\mathbf{k}}_{n}+\mathbf{H}\right) \cdot \nabla\right) \boldsymbol{\eta} \cdot \widehat{\mathbf{k}}_{n} d x+\lambda_{n} \int_{\Omega}\left(\widehat{\mathbf{k}}_{n} \cdot \nabla\right) \boldsymbol{\eta} \cdot \mathbf{H} d x+\lambda_{n} \int_{\Omega}(\mathbf{H} \cdot \nabla) \boldsymbol{\eta} \cdot \mathbf{H} d x \\
& \quad-\lambda_{n} \int_{\Omega}(\mathbf{U} \cdot \nabla) \boldsymbol{\eta} \cdot \mathbf{U} d x=\int_{\Omega} \hat{p}_{n} \operatorname{div} \boldsymbol{\eta} d x, \quad \forall \boldsymbol{\eta} \in W_{0}^{1,2}(\Omega) .
\end{aligned}
$$

Let $\widehat{\mathbf{u}}_{n}=\widehat{\mathbf{w}}_{n}+\mathbf{U}, \widehat{\mathbf{h}}_{n}=\widehat{\mathbf{k}}_{n}+\mathbf{H}$. Then identity (3.9) reduces to

$$
\begin{aligned}
\int_{\Omega} \nabla \widehat{\mathbf{u}}_{n} \cdot & \nabla \boldsymbol{\eta} d x-\int_{\Omega} \hat{p}_{n} \operatorname{div} \boldsymbol{\eta} d x=-\lambda_{n} \int_{\Omega}\left(\widehat{\mathbf{u}}_{n} \cdot \nabla\right) \widehat{\mathbf{u}}_{n} \cdot \boldsymbol{\eta} d x \\
& +\lambda_{n} \int_{\Omega}\left(\widehat{\mathbf{h}}_{n} \cdot \nabla\right) \widehat{\mathbf{h}}_{n} \cdot \boldsymbol{\eta} d x+\lambda_{n} \int_{\Omega}(\nabla \times \mathbf{f}) \cdot \boldsymbol{\eta} d x, \quad \forall \boldsymbol{\eta} \in W_{0}^{1,2}(\Omega) .
\end{aligned}
$$

Thus $\left(\widehat{\mathbf{u}}_{n}, \widehat{\mathbf{h}}_{n}, \hat{p}_{n}\right)$ might be considered as a weak solution to the Stokes problem

$$
\begin{cases}-\Delta \widehat{\mathbf{u}}_{n}+\nabla \hat{p}_{n}=-\lambda_{n}\left(\widehat{\mathbf{u}}_{n} \cdot \nabla\right) \widehat{\mathbf{u}}_{n}+\lambda_{n}\left(\widehat{\mathbf{h}}_{n} \cdot \nabla\right) \widehat{\mathbf{h}}_{n}+\lambda_{n} \nabla \times \mathbf{f}:=\mathbf{F}_{n} & \text { in } \Omega, \\ -\Delta \widehat{\mathbf{h}}_{n}=-\lambda_{n}\left(\widehat{\mathbf{u}}_{n} \cdot \nabla\right) \widehat{\mathbf{h}}_{n}+\lambda_{n}\left(\widehat{\mathbf{h}}_{n} \cdot \nabla\right) \widehat{\mathbf{u}}_{n}+\nabla \times \mathbf{g}:=\mathbf{H}_{n} & \text { in } \Omega, \\ \operatorname{div} \widehat{\mathbf{u}}_{n}=\operatorname{div} \widehat{\mathbf{h}}_{n}=0 & \text { in } \Omega, \\ \widehat{\mathbf{u}}_{n}=\mathbf{a}, \widehat{\mathbf{h}}_{n}=\mathbf{b} & \text { on } \partial \Omega .\end{cases}
$$

Obviously, $\mathbf{F}_{n}, \mathbf{H}_{n} \in L^{3 / 2}(\Omega)$ and

$$
\begin{aligned}
&\left\|\mathbf{F}_{n}\right\|_{L^{3 / 2}(\Omega)} \leq c\left\|\left(\widehat{\mathbf{u}}_{n} \cdot \nabla\right) \widehat{\mathbf{u}}_{n}\right\|_{L^{3 / 2}(\Omega)}+c\left\|\left(\widehat{\mathbf{h}}_{n} \cdot \nabla\right) \widehat{\mathbf{h}}_{n}\right\|_{L^{3 / 2}(\Omega)}+\|\nabla \times \mathbf{f}\|_{L^{3 / 2}(\Omega)} \\
& \leq c\left\|\widehat{\mathbf{u}}_{n}\right\|_{L^{6}(\Omega)}\left\|\nabla \widehat{\mathbf{u}}_{n}\right\|_{L^{2}(\Omega)}+c\left\|\widehat{\mathbf{h}}_{n}\right\|_{L^{6}(\Omega)}\left\|\nabla \widehat{\mathbf{h}}_{n}\right\|_{L^{2}(\Omega)}+\|\mathbf{f}\|_{W_{0}^{1,2}(\Omega)} \\
& \leq c\left(\left\|\widehat{\mathbf{w}}_{n}\right\|_{H(\Omega)}^{2}+\left\|\widehat{\mathbf{k}}_{n}\right\|_{H(\Omega)}^{2}+\|\mathbf{a}\|_{W^{1 / 2,2}(\partial \Omega)}^{2}+\|\mathbf{b}\|_{W^{1 / 2,2}(\partial \Omega)}^{2}\right)+\|\mathbf{f}\|_{W_{0}^{1,2}(\Omega)}, \\
&\left\|\mathbf{H}_{n}\right\|_{L^{3 / 2}(\Omega)} \leq c\left\|\left(\widehat{\mathbf{u}}_{n} \cdot \nabla\right) \widehat{\mathbf{h}}_{n}\right\|_{L^{3 / 2}(\Omega)}+c\left\|\left(\widehat{\mathbf{h}}_{n} \cdot \nabla\right) \widehat{\mathbf{u}}_{n}\right\|_{L^{3 / 2}(\Omega)}+\|\nabla \times \mathbf{g}\|_{L^{3 / 2}(\Omega)} \\
& \leq c\left(\left\|\widehat{\mathbf{w}}_{n}\right\|_{H(\Omega)}^{2}+\left\|\widehat{\mathbf{k}}_{n}\right\|_{H(\Omega)}^{2}+\|\mathbf{a}\|_{W^{1 / 2,2}(\partial \Omega)}^{2}+\|\mathbf{b}\|_{W^{1 / 2,2}(\partial \Omega)}^{2}\right)+\|\mathbf{g}\|_{W_{0}^{1,2}(\Omega)},
\end{aligned}
$$

where $c$ is independent of $n$. By the well-known regularity results for the Stokes system (see [10, Theorem IV.6.1]), we have $\widehat{\mathbf{u}}_{n}, \widehat{\mathbf{h}}_{n} \in W^{2,3 / 2}(\Omega), \hat{p}_{n} \in W^{1,3 / 2}(\Omega)$, and also the estimate

$$
\begin{aligned}
& \left\|\widehat{\mathbf{u}}_{n}\right\|_{W^{2,3 / 2}(\Omega)}+\left\|\hat{p}_{n}\right\|_{W^{1,3 / 2}(\Omega)} \leq c\left(\left\|\mathbf{F}_{n}\right\|_{L^{3 / 2}(\Omega)}+\|\mathbf{a}\|_{W^{3 / 2,2}(\partial \Omega)}\right) \\
\leq & c\left(\left\|\widehat{\mathbf{w}}_{n}\right\|_{H(\Omega)}^{2}+\left\|\widehat{\mathbf{k}}_{n}\right\|_{H(\Omega)}^{2}+\|(\mathbf{a}, \mathbf{b})\|_{W^{3 / 2,2}(\partial \Omega)}^{2}+\|(\mathbf{a}, \mathbf{b})\|_{W^{3 / 2,2}(\partial \Omega)}+\|\mathbf{f}\|_{W_{0}^{1,2}(\Omega)}\right), \\
& \left\|\widehat{\mathbf{h}}_{n}\right\|_{W^{2,3 / 2}(\Omega)} \leq c\left(\left\|\mathbf{H}_{n}\right\|_{L^{3 / 2}(\Omega)}+\|\mathbf{b}\|_{W^{3 / 2,2}(\partial \Omega)}\right) \\
\leq & c\left(\left\|\widehat{\mathbf{w}}_{n}\right\|_{H(\Omega)}^{2}+\left\|\widehat{\mathbf{k}}_{n}\right\|_{H(\Omega)}^{2}+\|(\mathbf{a}, \mathbf{b})\|_{W^{3 / 2,2}(\partial \Omega)}^{2}+\|(\mathbf{a}, \mathbf{b})\|_{W^{3 / 2,2}(\partial \Omega)}+\|\mathbf{g}\|_{W_{0}^{1,2}(\Omega)}\right) .
\end{aligned}
$$


Denote $\mathbf{u}_{n}=J_{n}^{-1} \widehat{\mathbf{u}}_{n}, \mathbf{h}_{n}=J_{n}^{-1} \widehat{\mathbf{h}}_{n}$ and $p_{n}=\lambda_{n}^{-1} J_{n}^{-2} \hat{p}_{n}$. Then

$$
\begin{array}{ll}
-\nu_{n} \Delta \mathbf{u}_{n}+\left(\mathbf{u}_{n} \cdot \nabla\right) \mathbf{u}_{n}+\nabla p_{n}=\left(\mathbf{h}_{n} \cdot \nabla\right) \mathbf{h}_{n}+\nabla \times \mathbf{f}_{n}, & \text { in } \Omega, \\
-\nu_{n} \Delta \mathbf{h}_{n}+\left(\mathbf{u}_{n} \cdot \nabla\right) \mathbf{h}_{n}-\left(\mathbf{h}_{n} \cdot \nabla\right) \mathbf{u}_{n}=\nabla \times \mathbf{g}_{n}, & \text { in } \Omega, \\
\operatorname{div} \mathbf{u}_{n}=\operatorname{div} \mathbf{h}_{n}=0, & \text { in } \Omega, \\
\mathbf{u}_{n}=\mathbf{a}_{n}, \quad \mathbf{h}_{n}=\mathbf{b}_{n}, & \text { on } \partial \Omega,
\end{array}
$$

where $\nu_{n}=\lambda_{n}^{-1} J_{n}^{-1}, \mathbf{f}_{n}=J_{n}^{-2} \mathbf{f}, \mathbf{g}_{n}=J_{n}^{-2} \mathbf{g}$ and $\mathbf{a}_{n}=J_{n}^{-1} \mathbf{a}, \mathbf{b}_{n}=J_{n}^{-1} \mathbf{b}$.

It follows from Equation (3.10) that

$$
\left\|p_{n}\right\|_{W^{1,3 / 2}(\Omega)} \leq \text { const. }
$$

Hence, from the sequence $\left\{p_{n_{l}}\right\}$, we can extract a subsequence, still denoted by $\left\{p_{n_{l}}\right\}$, which converges weakly in $W^{1,3 / 2}(\Omega)$ to some function $p \in W^{1,3 / 2}(\Omega)$. Let $\varphi \in C_{0}^{\infty}(\Omega)$. Taking $\boldsymbol{\eta}=J_{n}^{-2} \boldsymbol{\varphi}$ in Equation (3.9) and letting $n_{l} \rightarrow \infty$, we get

$$
-\lambda_{0} \int_{\Omega}(\mathbf{w} \cdot \nabla) \boldsymbol{\varphi} \cdot \mathbf{w} d x+\lambda_{0} \int_{\Omega}(\mathbf{k} \cdot \nabla) \boldsymbol{\varphi} \cdot \mathbf{k} d x=\lambda_{0} \int_{\Omega} p \operatorname{div} \boldsymbol{\varphi} d x \quad \forall \varphi \in C_{0}^{\infty}(\Omega) .
$$

Integrating by parts in the last equality, we derive

$$
\lambda_{0} \int_{\Omega}[(\mathbf{w} \cdot \nabla) \mathbf{w}-(\mathbf{k} \cdot \nabla) \mathbf{k}] \cdot \varphi d x=-\lambda_{0} \int_{\Omega} \nabla p \cdot \varphi d x \quad \forall \varphi \in C_{0}^{\infty}(\Omega) .
$$

Hence, the pair $(\mathbf{w}, \mathbf{k}, p)$ satisfies, for almost all $x \in \Omega$, the inviscid, nonresistive MHD equations

$$
\begin{cases}(\mathbf{w} \cdot \nabla) \mathbf{w}+\nabla p=(\mathbf{k} \cdot \nabla) \mathbf{k}, & \text { in } \Omega, \\ (\mathbf{w} \cdot \nabla) \mathbf{k}-(\mathbf{k} \cdot \nabla) \mathbf{w}=0, & \text { in } \Omega, \\ \operatorname{div} \mathbf{w}=\operatorname{div} \mathbf{k}=0, & \text { in } \Omega, \\ \mathbf{w}=\mathbf{k}=0, & \text { on } \partial \Omega .\end{cases}
$$

We summarize the above results as follows.

LEMMA 3.1. Assume that $\Omega \subset \mathbb{R}^{3}$ is a bounded axially symmetric domain of type (1.1) with $C^{2}$-smooth boundary $\partial \Omega,(\nabla \times \mathbf{f}, \nabla \times \mathbf{g}) \in W_{A S}^{1,2}(\Omega) \times W_{A \text { SoS }}^{1,2}(\Omega),(\mathbf{a}, \mathbf{b}) \in$ $W_{A S}^{3 / 2,2}(\partial \Omega) \times W_{A S o S}^{3 / 2,2}(\partial \Omega)$ are axially symmetric, and $\mathbf{a}$ and $\mathbf{b}$ satisfy conditions (1.3)(1.4). If the assertion of Theorem 1.1 is false, then there exist $\mathbf{w}, \mathbf{k}, p$ with the following properties:

(IMHD-AX) The axially symmetric functions $(\mathbf{w}, \mathbf{k}) \in H_{A S}(\Omega) \times H_{A S o S}(\Omega), p \in$ $W_{A S}^{1,3 / 2}(\Omega)$ satisfy the inviscid, nonresistive $M H D$ system (3.14) and Equation (3.7)

(MHD-AX) There exist a sequence of axially symmetric functions $\mathbf{u}_{n} \in W_{A S}^{1,2}(\Omega), \mathbf{h}_{n} \in$ $W_{A S o S}^{1,2}(\Omega), p_{n} \in W_{A S}^{1,3 / 2}(\Omega)$ and numbers $\nu_{n} \rightarrow 0+, \lambda_{n} \rightarrow \lambda_{0} \in(0,1]$ such that the norms $\left\|\mathbf{u}_{n}\right\|_{W^{1,2}(\Omega)}+\left\|\mathbf{h}_{n}\right\|_{W^{1,2}(\Omega)}$ and $\left\|p_{n}\right\|_{W^{1,3 / 2}(\Omega)}$ are uniformly bounded, the pair $\left(\mathbf{u}_{n}, \mathbf{h}_{n}, p_{n}\right)$ satisfies Equation (3.12), and

$$
\begin{aligned}
& \left\|\nabla \mathbf{u}_{n}\right\|_{L^{2}(\Omega)}+\left\|\nabla \mathbf{h}_{n}\right\|_{L^{2}(\Omega)} \rightarrow 1, \\
& \mathbf{u}_{n} \rightarrow \mathbf{w}, \quad \mathbf{h}_{n} \rightarrow \mathbf{k} \quad \text { in } W^{1,2}(\Omega), \quad p_{n} \rightarrow p \quad \text { in } W^{1,3 / 2}(\Omega) .
\end{aligned}
$$

Moreover, $\left(\mathbf{u}_{n}, \mathbf{h}_{n}\right) \in W_{l o c}^{3,2}(\Omega)$ and $p_{n} \in W_{l o c}^{2,2}(\Omega)$. 
Assume that

$$
\begin{array}{ll}
\Gamma_{j} \cap O_{x_{3}} \neq \emptyset, & j=0, \ldots, M^{\prime}, \\
\Gamma_{j} \cap O_{x_{3}}=\emptyset, & j=M^{\prime}+1, \ldots, N .
\end{array}
$$

Let $P_{+}=\left\{\left(0, x_{2}, x_{3}\right): x_{2}>0, x_{3} \in \mathbb{R}\right\}, \mathcal{D}=\Omega \cap P_{+}$. Obviously, on $P_{+}$, the coordinates $x_{2}, x_{3}$ coincide with the coordinates $r, z$. For a set $A \subset \mathbb{R}^{3}$, put $\breve{A}:=A \cap P_{+}$, and for $B \subset P_{+}$denote by $\tilde{B}$ the set in $\mathbb{R}^{3}$ obtained by rotation of $B$ around the $O_{z}$-axis. Then $\left(S_{1}\right) \mathcal{D}$ is a bounded plane domain with Lipschitz boundary. Moreover, $\breve{\Gamma}_{j}$ is a connected set for every $j=0, \ldots, N$. In other words, $\left\{\breve{\Gamma}_{j}: j=0, \ldots, N\right\}$ coincides with the family of all connected components of the set $P_{+} \cap \partial \mathcal{D}$.

Hence $\mathbf{w}, \mathbf{k}$, and $p$ satisfy the following system in the plan domain $\mathcal{D}$ :

$$
\left\{\begin{array}{l}
w_{r} \partial_{r} w_{r}+w_{z} \partial_{z} w_{r}-\frac{w_{\theta}^{2}}{r}+\partial_{r} p=-\frac{k_{\theta}^{2}}{r} \\
w_{r} \partial_{r} w_{\theta}+w_{z} \partial_{z} w_{\theta}+\frac{w_{r} w_{\theta}}{r}=0 \\
w_{r} \partial_{r} w_{z}+w_{z} \partial_{z} w_{z}+\partial_{z} p=0 \\
w_{r} \partial_{r} k_{\theta}+w_{z} \partial_{z} k_{\theta}-\frac{w_{r} k_{\theta}}{r}=0 \\
\partial_{r}\left(r w_{r}\right)+\partial_{z}\left(r w_{z}\right)=0
\end{array}\right.
$$

These equations are satisfied for almost all $x \in \mathcal{D}$ and

$$
\mathbf{w}(x)=\mathbf{k}(x)=0 \quad \text { for } \mathcal{H}^{1} \text {-almost all } x \in P_{+} \cap \partial \mathcal{D} .
$$

We have the following integral estimates: $\mathbf{w}, \mathbf{k} \in W_{\text {loc }}^{1,2}(\mathcal{D})$,

$$
\int_{\mathcal{D}}\left(|\nabla \mathbf{w}(r, z)|^{2}+|\nabla \mathbf{k}(r, z)|^{2}\right) r d r d z<\infty
$$

and, by the Sobolev embedding theorem for three-dimensional domains, $\mathbf{w}, \mathbf{k} \in L^{6}(\Omega)$, i.e.,

$$
\int_{\mathcal{D}}\left(|\mathbf{w}(r, z)|^{6}+|\mathbf{k}(r, z)|^{6}\right) r d r d z<\infty
$$

Also, the condition $\nabla p \in L^{3 / 2}(\Omega)$ can be written as

$$
\int_{\mathcal{D}}|\nabla p(r, z)|^{3 / 2} r d r d z<\infty
$$

Denote by $\Phi=p+\frac{|\mathbf{w}|^{2}}{2}+\frac{|\mathbf{k}|^{2}}{2}$ the total head pressure corresponding to the solution $(\mathbf{w}, \mathbf{k}, p)$. Obviously,

$$
\int_{\mathcal{D}} r|\nabla \Phi(r, z)|^{3 / 2} d r d z<\infty
$$

Hence,

$$
\Phi \in W^{1,3 / 2}\left(\mathcal{D}_{\epsilon}\right) \quad \forall \epsilon>0 .
$$

We also have the important Bernoulli's law: for almost all $x \in \mathcal{D}$,

$$
\left(w_{r} \partial_{r}+w_{z} \partial_{z}\right) \Phi=0
$$


3.2. Some results on inviscid MHD equations. Since $\mathbf{w}$ and $\mathbf{k}$ satisfy Equation (3.14), $\mathbf{w}=\mathbf{k} \equiv 0$ on $\partial \Omega$, and $\nabla p \in L^{3 / 2}(\Omega)$ : then one can follow [1] and [12] to prove the following statement.

LEMMA 3.2. If (IMHD-AX) are satisfied, then

$$
\forall j \in\{0,1, \ldots, N\} \quad \exists p_{j} \in \mathbb{R}: \quad p(x) \equiv p_{j} \quad \text { for } \mathcal{H}^{2} \text {-almost all } x \in \Gamma_{j} .
$$

In particular, by axial symmetry,

$$
p(x) \equiv p_{j} \quad \text { for } \mathcal{H}^{1} \text {-almost all } x \in \breve{\Gamma}_{j}
$$

We need a weak version of Bernoulli's law for a Sobolev solution $(\mathbf{w}, \mathbf{k}, p)$ to the inviscid MHD Equations (3.16).

From the last equality in Equations (3.16) and from Equation (3.18), it follows that there exists a stream function $\psi \in W_{l o c}^{2,2}(\mathcal{D})$ such that

$$
\frac{\partial \psi}{\partial r}=-r w_{z}, \quad \frac{\partial \psi}{\partial z}=r w_{r}
$$

Fix a point $x_{*} \in \mathcal{D}$. For $\epsilon>0$, denote by $\mathcal{D}_{\epsilon}$ the connected component of $\mathcal{D} \cap\{(r, z)$ : $r>\epsilon\}$ containing $x_{*}$. Since

$$
\psi \in W^{2,2}\left(\mathcal{D}_{\epsilon}\right) \quad \forall \epsilon>0,
$$

by the Sobolev embedding theorem, $\psi \in C\left(\overline{\mathcal{D}_{\epsilon}}\right)$. Hence $\psi$ is continuous at points of $\overline{\mathcal{D}} \backslash O_{z}=\overline{\mathcal{D}} \backslash\{(0, z): z \in \mathbb{R}\}$. By the definition of $\psi$ and since $\mathbf{w}=\mathbf{k} \equiv 0$ on $\partial \Omega$, we see that all the boundary components are level sets of $\psi$.

LEMMA 3.3. If (IMHD-AX) are satisfied, then there exist constants $\xi_{0}, \ldots, \xi_{N} \in \mathbb{R}$ such that $\psi(x) \equiv \xi_{j}$ on each curve $\breve{\Gamma}_{j}, j=0, \ldots, N$.

Proof. By virtue of Equations (3.17) and (3.26), we have $\nabla \psi(x)=0$ for $\mathcal{H}^{1}$-almost all $x \in \partial \mathcal{D} \backslash O_{z}$. Then the Morse-Sard property (see [3]) implies that

$$
\text { for any connected set } C \subset \partial \mathcal{D} \backslash O_{z}, \quad \exists \alpha=\alpha(C) \in \mathbb{R}: \psi(x) \equiv \alpha \quad \forall x \in C .
$$

Hence since $\breve{\Gamma}_{j}$ are connected, the lemma follows.

By the properties of Sobolev functions $\mathbf{w}, \mathbf{k}, \psi, \Phi$ (see [5]), we get the following.

LEMMA 3.4. If conditions (IMHD-AX) hold, then there exists a set $A_{\mathbf{w}} \subset \overline{\mathcal{D}}$ such that

(i) $\mathcal{H}^{1}\left(A_{\mathbf{w}}\right)=0$;

(ii) for all $x=(r, z) \in \mathcal{D} \backslash A_{\mathbf{w}}$,

$\lim _{\rho \rightarrow 0} f_{B_{\rho}(x)}|\mathbf{w}(y)-\mathbf{w}(x)|^{2} d y=\lim _{\rho \rightarrow 0} f_{B_{\rho}(x)}|\mathbf{k}(y)-\mathbf{k}(x)|^{2} d y=\lim _{\rho \rightarrow 0} f_{B_{\rho}(x)}|\Phi(y)-\Phi(x)|^{2} d y=0 ;$

moreover, the function $\psi$ is differentiable at $x$, and

$$
\nabla \psi(x)=\left(-r w_{z}(x), r w_{r}(x)\right) ; \text { and }
$$

(iii) for every $\epsilon>0$, there exists a set $U \subset \mathbb{R}^{2}$ with $\mathcal{H}_{\infty}^{1}(U)<\epsilon, A_{\mathbf{w}} \subset U$ and such that the functions $\mathbf{w}, \mathbf{k}, \Phi$ are continuous on $\overline{\mathcal{D}} \backslash\left(U \cup O_{z}\right)$. 
Then one can mimic the proof in [15] to establish the following weak version of Bernoulli's law.

Lemma 3.5 (Bernoulli's Law). Let conditions (IMHD-AX) be valid, and let $A_{\mathbf{w}}$ be a set from Lemma 3.4. For any compact connected set $K \subset \overline{\mathcal{D}} \backslash O_{z}$, the following property holds: if

$$
\left.\psi\right|_{K}=\text { const, }
$$

then

$$
\Phi\left(x_{1}\right)=\Phi\left(x_{2}\right) \quad \text { for all } x_{1}, x_{2} \in K \backslash A_{\mathbf{w}} .
$$

In particular, we can denote by $\Phi(K)$ the uniform constant $c \in \mathbb{R}$ such that $\Phi(x)=c$ for all $x \in K \backslash A_{\mathrm{w}}$ for any compact set $K \subset \overline{\mathcal{D}} \backslash O_{z}$ with $\psi_{K}=$ const. Moreover, $\Phi$ has some continuity properties when $K$ approaches the singularity axis $O_{z}$.

LEMMA 3.6. Assume that conditions (IMHD-AX) are satisfied. Let $K_{i}$ be a sequence of compact sets with the following properties: $K_{i} \subset \overline{\mathcal{D}} \backslash O_{z},\left.\psi\right|_{K_{i}}=$ const, and $\lim _{i \rightarrow \infty} \inf _{(r, z) \in K_{i}} r=0, \liminf _{i \rightarrow \infty} \sup _{(r, z) \in K_{i}} r>0$. Then $\Phi\left(K_{i}\right) \rightarrow p_{0}$ as $i \rightarrow \infty$.

LEMMA 3.7. If conditions (IMHD-AX) are satisfied, then $p_{0}=\cdots=p_{M^{\prime}}$, where $p_{j}$ are the constants from Lemma 3.2.

Heuristically, one can imagine that the axis $O z$ is an "almost" streamline. By Lemma 3.5, all the boundary components that intersects with the symmetry axis should share the same total head pressure $\Phi$, which immediately implies Lemma 3.7. Since the proof of lemmas 3.2-3.7 are quite similar to the proofs in [15], we omit the details.

3.3. Obtaining a contradiction. We consider three possible cases.

(a) The maximum of $\Phi$ is attained on the boundary component intersecting the symmetry axis:

$$
p_{0}=\max _{j=0, \ldots, N} p_{j}=\sup _{x \in \Omega} \Phi(x) .
$$

(b) The maximum of $\Phi$ is attained on a boundary component that does not intersect the symmetry axis:

$$
p_{0}<p_{N}=\max _{j=0, \ldots, N} p_{j}=\sup _{x \in \Omega} \Phi(x) .
$$

(c) The maximum of $\Phi$ is not attained on $\partial \Omega$ :

$$
\max _{j=0, \ldots, N} p_{j}<\sup _{x \in \Omega} \Phi(x) .
$$

3.3.1. The case $\sup _{x \in \Omega} \Phi(x)=p_{0}$. Adding a constant to the pressure $p$, we can assume that

$$
p_{0}=\sup _{x \in \Omega} \Phi(x)=0
$$

Since the identity $p_{0}=p_{1}=\cdots=p_{N}$ is impossible, we have that $p_{j}<0$ for some $j \in\left\{M^{\prime}+1, N\right\}$. Recall that, by Lemma $3.7, p_{0}=p_{1}=\cdots=p_{M^{\prime}}=0$. From Equation $(3.14)_{1}$, we obtain

$$
0=x \cdot \nabla p(x)+x \cdot(\mathbf{w}(x) \cdot \nabla) \mathbf{w}(x)-x \cdot(\mathbf{k}(x) \cdot \nabla) \mathbf{k}(x)
$$




$$
\begin{aligned}
& =\operatorname{div}[x p(x)+(\mathbf{w}(x) \cdot x) \mathbf{w}(x)-(\mathbf{k}(x) \cdot x) \mathbf{k}(x)]-p(x) \operatorname{div} x-|\mathbf{w}(x)|^{2}+|\mathbf{k}(x)|^{2} \\
& =\operatorname{div}[x p(x)+(\mathbf{w}(x) \cdot x) \mathbf{w}(x)-(\mathbf{k}(x) \cdot x) \mathbf{k}(x)]-3 \Phi(x)+\frac{1}{2}|\mathbf{w}(x)|^{2}+\frac{5}{2}|\mathbf{k}(x)|^{2} .
\end{aligned}
$$

Integrating it over $\partial \Omega$ and using Equation (3.33), we derive a contradiction as follows:

$$
\begin{aligned}
0 & \geq \int_{\Omega}\left[3 \Phi(x)-\frac{1}{2}|\mathbf{w}(x)|^{2}-\frac{5}{2}|\mathbf{k}(x)|^{2}\right] d x=\int_{\partial \Omega} p(x)(x \cdot \mathbf{n}) d s=\sum_{j=0}^{N} p_{j} \int_{\Gamma_{j}}(x \cdot \mathbf{n}) d s \\
& =\sum_{j=1}^{N} p_{j} \int_{\Omega_{j}} \operatorname{div} x d x=-3 \sum_{j=1}^{N} p_{j}\left|\Omega_{j}\right|>0 .
\end{aligned}
$$

Hence, we exclude the first case.

3.3.2. The case: $p_{0}<p_{N}=\sup _{x \in \bar{\Omega}} \Phi(x)$. We may assume that the maximum value is zero:

$$
p_{0}<p_{N}=\max _{j=0, \ldots, N} p_{j}=\sup _{x \in \Omega} \Phi(x)=0 .
$$

Then $p_{0}=\cdots=p_{M^{\prime}}<0$. so that

Change (if necessary) the numbering of the boundary components $\Gamma_{M^{\prime}+1}, \ldots, \Gamma_{N-1}$

$$
\begin{array}{r}
p_{j}<0, j=0, \ldots, M, M \geq M^{\prime}, \\
p_{M+1}=\cdots=p_{N}=0 .
\end{array}
$$

To remove a neighborhood of the singularity line $O_{z}$ from our consideration, we take $r_{0}>0$ such that the open set $\mathcal{D}_{\epsilon}=\{(r, z) \in \mathcal{D}: r>\epsilon\}$ is connected for every $\epsilon \leq r_{0}$ (i.e., $\mathcal{D}_{\epsilon}$ is a domain), and

$$
\begin{aligned}
& \breve{\Gamma}_{j} \subset \overline{\mathcal{D}_{r_{0}}} \text { and } \quad \inf _{(r, z) \in \breve{\Gamma}_{j}} r \geq 2 r_{0}, \quad j=M^{\prime}+1, \ldots, N, \\
& \breve{\Gamma}_{j} \cap \overline{\mathcal{D}_{\epsilon}} \text { is a connected set and } \sup _{(r, z) \in \breve{\Gamma}_{j} \cap \overline{\mathcal{D}}_{\epsilon}} r \geq 2 r_{0}, j=0, \ldots, M^{\prime}, \epsilon \in\left(0, r_{0}\right] .
\end{aligned}
$$

Let a set $C \subset \overline{\mathcal{D}_{\epsilon}}$ separate $\breve{\Gamma}_{i}$ and $\breve{\Gamma}_{j}$ in $\mathcal{D}_{\epsilon}$ for some different indexes $i, j \in\{0, \ldots, N\}$; i.e., $\breve{\Gamma}_{i} \cap \overline{\mathcal{D}_{\epsilon}}$ and $\breve{\Gamma}_{j} \cap \overline{\mathcal{D}_{\epsilon}}$ lie in different connected components of $\overline{\mathcal{D}_{\epsilon}} \backslash C$. Obviously, for $\epsilon \in\left(0, r_{0}\right]$, there exists a constant $\delta(\epsilon)>0$ (not depending on $i, j, C$ ) such that the uniform estimate $\sup _{(r, z) \in C} r \geq \delta(\epsilon)$ holds. Moreover, the function $\delta(\epsilon)$ is nondecreasing. In particular,

$$
\delta(\epsilon) \geq \delta\left(r_{0}\right), \quad \epsilon \in\left(0, r_{0}\right]
$$

In the following, we will construct an appropriate integration domain by using the level sets of $\Phi$ and $\Phi_{n}$. We need some information concerning the behavior of the limit total head pressure $\Phi$ on stream lines. Following [16] and [19], we introduce some facts of topology. By continuum we mean a compact connected set. We understand connectedness in the sense of general topology. A subset of a topological space is called an $\operatorname{arc}$ if it is homeomorphic to the unit interval $[0,1]$. Let $Q=[0,1] \times[0,1]$ be a square in $\mathbb{R}^{2}$, and let $f$ be a continuous function on $Q$. Denote by $E_{t}$ a level set of the function $f$, i.e., $E_{t}=\{x \in Q: f(x)=t\}$. A connected component $K$ of the level set $E_{t}$ containing 
a point $x_{0}$ is a maximal connected subset of $E_{t}$ containing $x_{0}$. By $T_{f}$ denote a family of all connected components of level sets of $f$.

We apply Kronrod's results to the stream function $\left.\psi\right|_{\overline{\mathcal{D}}_{\epsilon}}, \epsilon \in\left(0, r_{0}\right]$. Accordingly, $T_{\psi, \epsilon}$ means the corresponding Kronrod tree for the restriction $\left.\psi\right|_{\overline{\mathcal{D}}}$. Define the total head pressure on the Kronrod tree $T_{\psi, \epsilon}$ as follows. Let $K \in T_{\psi, \epsilon}$ with diam $K>0$. Take any $x \in K \backslash A_{\mathbf{w}}$ and put $\Phi(K)=\Phi(x)$. By the Bernoulli's Law in Lemma 3.5, the value $\Phi(K)$ is independent of the choice $x \in K \backslash A_{\mathbf{w}}$. Then $\Phi$ has the following continuity properties on stream lines.

Lemma 3.8 (See [16, Lemma 3.5]). Let $A, B \in T_{\psi, \epsilon}$, where $\epsilon \in\left(0, r_{0}\right]$, diam $A>0$, and diam $B>0$. Consider the corresponding arc $[A, B] \subset T_{\psi, \epsilon}$ joining $A$ to $B$. Then the restriction $\left.\Phi\right|_{[A, B]}$ is a continuous function.

Denote by $B_{0}^{\epsilon}, \ldots, B_{N}^{\epsilon}$ the elements of $T_{\psi, \epsilon}$ such that $B_{j}^{\epsilon} \supset \breve{\Gamma}_{j} \cap \overline{\mathcal{D}_{\epsilon}}, j=0, \ldots, M^{\prime}$, and $B_{j}^{\epsilon} \supset \breve{\Gamma}_{j}, j=M^{\prime}+1, \ldots, N$. By construction, $\Phi\left(B_{j}^{\epsilon}\right)<0$ for $j=0, \ldots, M$, and $\Phi\left(B_{j}^{\epsilon}\right)=0$ for $j=M+1, \ldots, N$. For $r>0$, let $L_{r}$ be the horizontal straight line $L_{r}=\{(r, z): z \in \mathbb{R}\}$. Then, similar to [16, Lemma 4.6], we can find $r_{*} \in\left(0, r_{0}\right]$ and $C_{j} \in\left[B_{j}^{r_{*}}, B_{N}^{r_{*}}\right], j=0, \ldots, M$, such that $\Phi\left(C_{j}\right)<0$ and $C \cap L_{r_{*}}=\emptyset$ for all $C \in\left[C_{j}, B_{N}^{r_{*}}\right]$.

We restrict our argument on the domain $\mathcal{D}_{r_{*}}$ and put $T_{\psi}=T_{\psi, r_{*}}$ and $B_{j}=B_{j}^{r_{*}}$. Since $\partial \mathcal{D}_{r_{*}} \subset B_{0} \cup \cdots \cup B_{N} \cup L_{r_{*}}$ and the set $\left\{B_{0}, \ldots, B_{N}\right\} \subset T_{\psi}$ is finite, we can change $C_{j}$ (if necessary) such that

$$
\begin{aligned}
& \forall j=0, \ldots, M, \quad C_{j} \in\left[B_{j}, B_{N}\right], \quad \Phi\left(C_{j}\right)<0, \\
& C \cap \partial \mathcal{D}_{r_{*}}=\emptyset \quad \forall C \in\left[C_{j}, B_{N}\right) .
\end{aligned}
$$

Observe that $\Gamma_{j} \cap L_{r_{*}} \neq \emptyset$ for $j=0, \ldots, M^{\prime}$. Therefore, if a cycle $C \in T_{\psi}$ separates $\Gamma_{N}$ from $\Gamma_{0}$ and $C \cap \partial \mathcal{D}_{r_{*}}=\emptyset$, then $C$ separates $\Gamma_{N}$ from $\Gamma_{j}$ for all $j=1, \ldots, M^{\prime}$. So we can take $C_{0}=\cdots=C_{M^{\prime}}$ and consider only the Kronrod $\operatorname{arcs}\left[C_{M^{\prime}}, B_{N}\right], \ldots,\left[C_{M}, B_{N}\right]$. Recall that a set $\mathcal{Z} \subset T_{\psi}$ has $T$-measure zero if $\mathcal{H}^{1}(\{\psi(C): C \in \mathcal{Z}\})=0$.

Lemma 3.9. For every $j=M^{\prime}, \ldots, M, T$-almost all $C \in\left[C_{j}, B_{N}\right]$ are $C^{1}$-curves homeomorphic to the circle. Moreover, there exists a subsequence $\Phi_{n_{l}}$ such that the sequence $\left.\Phi_{n_{l}}\right|_{C}$ converges to $\left.\Phi\right|_{C}$ uniformly $\left.\left.\Phi_{n}\right|_{C} \rightrightarrows \Phi\right|_{C}$ on T-almost all cycles $C \in\left[C_{j}, B_{N}\right]$.

Without loss of generality, we assume that the subsequence $\Phi_{n_{l}}$ coincides with $\Phi_{n}$. Besides, cycles satisfying the assertion of Lemma 3.9 will be called regular cycles. From Lemma 3.9 and [16, Lemma 3.6], we can conclude that

$$
\mathcal{H}^{1}\left(\left\{\Phi(C): C \in\left[C_{j}, B_{N}\right] \text { and } C \text { is not a regular cycle }\right\}\right)=0, j=M^{\prime}, \ldots, M .
$$

Setting $\alpha=\max _{j=M^{\prime}, \ldots, M} \min _{C \in\left[C_{j}, B_{N}\right]} \Phi(C)$, by (3.40), $\alpha<0$. By Equation (3.42), we can find a sequence of positive values $t_{i} \in(0,-\alpha), i \in \mathbb{N}$ with $t_{i+1}=\frac{1}{2} t_{i}$ such that the implication

$$
\Phi(C)=-t_{i} \Rightarrow C \text { is a regular cycle }
$$

holds for every $j=M^{\prime}, \ldots, M$ and for all $C \in\left[C_{j}, B_{N}\right]$. Consider the natural order on the arc $\left[C_{j}, B_{N}\right]$, namely, $C^{\prime}<C^{\prime \prime}$ if $C^{\prime \prime}$ is closer to $B_{N}$ than $C^{\prime}$. For $j=M^{\prime}, \ldots, M$ and $i \in \mathbb{N}$, put

$$
A_{i}^{j}=\max \left\{C \in\left[C_{j}, B_{N}\right]: \Phi(C)=-t_{i}\right\} .
$$

Then each $A_{i}^{j}$ is a regular cycle and $A_{i}^{j} \subset \mathcal{D}_{r_{*}}$. In particular, for each $i \in \mathbb{N}$, the compact set $\cup_{j=M^{\prime}}^{M} A_{i}^{j}$ is separated from $\partial \mathcal{D}_{r_{*}}$ and $\operatorname{dist}\left(\cup_{j=M^{\prime}}^{M} A_{i}^{j}, \partial \mathcal{D}_{r_{*}}\right)>0$. Then for each $i$ 
and for sufficiently small $h>0$, we have the inclusion $\left\{x \in \mathcal{D}_{r_{*}}: \operatorname{dist}\left(x, \breve{\Gamma}_{N}\right)<h\right\} \subset \mathcal{D}_{r_{*}} \backslash$ $\left(\cup_{j=M^{\prime}}^{M} A_{i}^{j}\right)$. Denote by $V_{i}$ the connected component of the open set $\mathcal{D}_{r_{*}} \backslash\left(\cup_{j=M^{\prime}}^{M} A_{i}^{j}\right)$ which encloses the set $\left\{x \in \mathcal{D}_{r_{*}}: \operatorname{dist}\left(x, \breve{\Gamma}_{N}\right)<h\right\}$. Then we have

$$
\left\{x \in \mathcal{D}_{r_{*}}: \operatorname{dist}\left(x, \breve{\Gamma}_{N}\right)<h\right\} \cap \partial V_{i}=A_{i}^{M^{\prime}} \cup \cdots \cup A_{i}^{M} .
$$

By the construction, the sequence of domains $V_{i}$ is decreasing, i.e., $V_{i} \supset V_{i+1}$. Hence, the sequence of sets $\left(\partial \mathcal{D}_{r_{*}}\right) \cap\left(\partial V_{i}\right)$ is nonincreasing. Every set $\left(\partial \mathcal{D}_{r_{*}}\right) \cap\left(\partial V_{i}\right)$ consists of several components $\breve{\Gamma}_{l}$ with $l>M$. Since there are only finitely many components $\Gamma_{l}$, we can conclude that, for sufficiently large $i$, the set $\left(\partial \mathcal{D}_{r_{*}}\right) \cap\left(\partial V_{i}\right)$ is independent of $i$. So we can assume that $\left(\partial \mathcal{D}_{r_{*}}\right) \cap\left(\partial V_{i}\right)=\breve{\Gamma}_{K} \cup \cdots \cup \breve{\Gamma}_{N}$, where $K \in\{M+1, \ldots, N\}$. Hence,

$$
\partial V_{i}=A_{i}^{M^{\prime}} \cup \cdots \cup A_{i}^{M} \cup \breve{\Gamma}_{K} \cup \cdots \cup \breve{\Gamma}_{N}
$$

By Lemma 3.9, we have the uniform convergence $\left.\Phi_{n}\right|_{A_{i}^{j}} \rightrightarrows \Phi\left(A_{i}^{j}\right)$ as $n \rightarrow \infty$. Then for each $i \in \mathbb{N}$ there exists $n_{i}$ such that for all $n \geq n_{i}$

$$
\left.\Phi_{n}\right|_{A_{i}^{j}}<-\frac{7}{8} t_{i},\left.\quad \Phi_{n}\right|_{A_{i+1}^{j}}>-\frac{5}{8} t_{i} \quad \forall j=M^{\prime}, \ldots, M
$$

Then

$$
\forall t \in\left[\frac{5}{8} t_{i}, \frac{7}{8} t_{i}\right] \quad \forall n \geq\left. n_{i} \quad \Phi_{n}\right|_{A_{i}^{j}}<-t,\left.\quad \Phi_{n}\right|_{A_{i+1}^{j}}>-t \quad \forall j=M^{\prime}, \ldots, M
$$

Accordingly, for $n \geq n_{i}$ and $t \in\left[\frac{5}{8} t_{i}, \frac{7}{8} t_{i}\right]$, we can define $W_{i n}^{j}(t)$ as the connected component of the open set $\left\{x \in V_{i} \backslash \overline{V_{i+1}}: \Phi_{n}(x)>-t\right\}$ with $\partial W_{i n}^{j}(t) \supset A_{i+1}^{j}$ and put

$$
W_{i n}(t)=\bigcup_{j=M^{\prime}}^{M} W_{i n}^{j}(t), \quad S_{i n}(t)=\left(\partial W_{i n}(t)\right) \cap\left(V_{i} \backslash \overline{V_{i+1}}\right) .
$$

By construction, $\Phi_{n} \equiv-t$ on $S_{i n}(t)$ and

$$
\partial W_{i n}(t)=S_{i n}(t) \cup A_{i+1}^{M^{\prime}} \cup \cdots \cup A_{i+1}^{M},
$$

and the set $S_{i n}(t)$ separates $A_{i}^{M^{\prime}} \cup \cdots \cup A_{i}^{M}$ from $A_{i+1}^{M^{\prime}} \cup \cdots \cup A_{i+1}^{M}$. Since $\Phi_{n} \in W_{l o c}^{2,2}(\Omega)$, by the Morse-Sard theorem, for almost all $t \in\left[\frac{5}{8} t_{i}, \frac{7}{8} t_{i}\right]$, the level set $S_{i n}(t)$ consists of finitely many $C^{1}$-cycles, and $\Phi_{n}$ is differentiable in the classical sense at every point $x \in$ $S_{i n}(t)$ with $\nabla \Phi_{n}(x) \neq 0$. We will say the values $t \in\left[\frac{5}{8} t_{i}, \frac{7}{8} t_{i}\right]$ having the above property are $(n, i)$-regular. Therefore, $\widetilde{S_{i n}}(t)$ is a finite union of smooth surfaces (tori), and by construction

$$
\int_{\widehat{S_{i n}}(t)} \nabla \Phi_{n} \cdot \mathbf{n} d S=-\int_{\widehat{S_{i n}}(t)}\left|\nabla \Phi_{n}\right| d S<0,
$$

where $\mathbf{n}$ is the unit outward normal vector to $\partial \widetilde{W_{i n}}(t)$.

For $h>0$, denote $\Gamma_{h}=\left\{x \in \Omega: \operatorname{dist}\left(x, \Gamma_{K} \cup \cdots \cup \Gamma_{N}\right)=h\right\}, \Omega_{h}=\left\{x \in \Omega: \operatorname{dist}\left(x, \Gamma_{K} \cup\right.\right.$ $\left.\left.\cdots \cup \Gamma_{N}\right)<h\right\}$. Since the distance function $\operatorname{dist}(x, \partial \Omega)$ is $C^{1}$-regular and the norm of its gradient is equal to one in the neighborhood of $\partial \Omega$, there is a constant $\delta_{0}>0$ such that, 
for every $h \leq \delta_{0}$, the set $\Gamma_{h}$ is a union of $N-K+1 C^{1}$-smooth surfaces homeomorphic to the torus, and

$$
\mathcal{H}^{2}\left(\Gamma_{h}\right) \leq c_{0} \quad \forall h \in\left(0, \delta_{0}\right]
$$

where the constant $c_{0}=3 \mathcal{H}^{2}\left(\Gamma_{K} \cup \cdots \cup \Gamma_{N}\right)$ is independent of $h$.

Lemma 3.10. For any $i \in \mathbb{N}$, there exists $n(i) \in \mathbb{N}$ such that, for every $n \geq n(i)$ and for almost all $t \in\left[\frac{5}{8} t_{i}, \frac{7}{8} t_{i}\right]$, the inequality

$$
\int_{\widetilde{S_{\text {in }}}(t)}\left|\nabla \Phi_{n}\right| d S \leq \mathcal{F} t
$$

holds with the constant $\mathcal{F}$ independent of $t, n$, and $i$.

Proof. By a direct calculation, Equation (3.16) implies

$$
\begin{aligned}
\nabla \Phi & =\nabla \frac{1}{2}|\mathbf{w}|^{2}-(\mathbf{w} \cdot \nabla) \mathbf{w}+\nabla \frac{1}{2}|\mathbf{k}|^{2}+(\mathbf{k} \cdot \nabla) \mathbf{k} \\
& =\left[\nabla \mathbf{w}-(\nabla \mathbf{w})^{T}\right] \cdot \mathbf{w}+\left[\nabla \mathbf{k}+(\nabla \mathbf{k})^{T}\right] \cdot \mathbf{k} .
\end{aligned}
$$

Since $\Phi \neq$ const on $\widetilde{V}_{i},(3.47)$ implies $\int_{\widetilde{V}_{i}}\left|\nabla \mathbf{w}-(\nabla \mathbf{w})^{T}\right|^{2}+\left|\nabla \mathbf{k}+(\nabla \mathbf{k})^{T}\right|^{2} d x>0$ for every $i$. Hence, from the weak convergence $\nabla \mathbf{u}_{n} \rightarrow \nabla \mathbf{w}$ and $\nabla \mathbf{h}_{n} \rightarrow \nabla \mathbf{k}$ in $L^{2}(\Omega)$ it follows that for any $i \in \mathbb{N}$, there exist constants $\epsilon_{i}>0, \delta_{i} \in\left(0, \delta_{0}\right)$ and $k_{i}^{\prime} \in \mathbb{N}$ such that

$$
\overline{\Omega_{\delta_{i}}} \cap \widetilde{A_{i}^{j}}=\overline{\Omega_{\delta_{i}}} \cap \widetilde{A_{i+1}^{j}}=\emptyset, \quad j=M^{\prime}, \ldots, M
$$

and for all $n \geq n_{i}^{\prime}$

$$
\int_{\widetilde{V_{i+1}} \backslash \Omega_{\delta_{i}}}\left(\left|\nabla \mathbf{u}_{n}-\left(\nabla \mathbf{u}_{n}\right)^{T}\right|^{2}+\left|\nabla \mathbf{h}_{n}+\left(\nabla \mathbf{h}_{n}\right)^{T}\right|^{2}\right) d x>\epsilon_{i}
$$

Fix $i \in \mathbb{N}$. We assume that $n \geq n_{i}$. Since we have removed a neighborhood of the singularity line $O_{z}$, we can use the Sobolev embedding theorem in the plane domain $\mathcal{D}_{r_{*}}$. The uniformly boundedness of $\left\|\Phi_{n}\right\|_{W^{1,3 / 2}\left(\mathcal{D}_{r_{*}}\right)}$ imply that the norm $\left\|\Phi_{n}\right\|_{L^{6}\left(\mathcal{D}_{r_{*}}\right)}$ and then $\left\|\Phi_{n} \nabla \Phi_{n}\right\|_{L^{6 / 5}\left(\mathcal{D}_{r_{*}}\right)}$ are also uniformly bounded. Finally, we have

$$
\left\|\Phi_{n} \nabla \Phi_{n}\right\|_{L^{6 / 5}\left(\widetilde{\mathcal{D}_{r_{*}}}\right)} \leq \text { const. }
$$

Fix a sufficiently small $\sigma>0$ (the exact value of $\sigma$ will be specified below), and take the parameter $\delta_{\sigma} \in\left(0, \delta_{i}\right]$ small enough to satisfy the following conditions:

$$
\begin{gathered}
\Omega_{\delta_{\sigma}} \cap \widetilde{A_{i}^{j}}=\Omega_{\delta_{\sigma}} \cap \widetilde{A_{i+1}^{j}}=\emptyset, \quad j=M^{\prime}, \ldots, M, \\
\int_{\Gamma_{h}} \Phi_{n}^{2} d S<\sigma^{2} \quad \forall h \in\left(0, \delta_{\sigma}\right] \quad \forall n \geq n^{\prime} .
\end{gathered}
$$

The last estimate follows from the identity $\left.\Phi\right|_{\Gamma_{K} \cup \ldots \cup \Gamma_{N}} \equiv 0$, the weak convergence $\Phi_{n} \rightarrow$ $\Phi$ in the space $W^{1,3 / 2}(\Omega)$, and (3.49).

By a direct calculation, (3.12) implies

$$
\begin{aligned}
\nabla \Phi_{n}= & -\nu_{n} \operatorname{curl} \text { curl } \mathbf{u}_{n}+\left[\nabla \mathbf{u}_{n}-\left(\nabla \mathbf{u}_{n}\right)^{T}\right] \cdot \mathbf{u}_{n} \\
& +\left[\nabla \mathbf{h}_{n}+\left(\nabla \mathbf{h}_{n}\right)^{T}\right] \cdot \mathbf{h}_{n}+\nabla \times \mathbf{f}_{n} .
\end{aligned}
$$


Then, using Stokes' theorem, we obtain

$$
\int_{S} \nabla \Phi_{n} \cdot \mathbf{n} d S=\int_{S}\left(\left[\nabla \mathbf{u}_{n}-\left(\nabla \mathbf{u}_{n}\right)^{T}\right] \cdot \mathbf{u}_{n}\right) \cdot \mathbf{n} d S+\int_{S}\left(\left[\nabla \mathbf{h}_{n}+\left(\nabla \mathbf{h}_{n}\right)^{T}\right] \cdot \mathbf{h}_{n}\right) \cdot \mathbf{n} d S .
$$

Now, fix a sufficiently small $\epsilon>0$. The exact value of $\epsilon$ will be specified below. For a given sufficiently large $n \geq n^{\prime}$, we follow the proof of [16, Lemma 3.8] to find a number $\overline{h_{n}} \in\left(0, \delta_{\sigma}\right)$ such that the estimates

$$
\begin{gathered}
\left|\int_{\Gamma_{\overline{h_{n}}}} \nabla \Phi_{n} \cdot \mathbf{n} d S\right| \leq 2 \int_{\Gamma_{\overline{h_{n}}}}\left(\left|\mathbf{u}_{n}\right| \cdot\left|\nabla \mathbf{u}_{n}\right|+\left|\mathbf{h}_{n}\right| \cdot\left|\nabla \mathbf{h}_{n}\right|\right) d S<\epsilon, \\
\int_{\Gamma_{\overline{h_{n}}}}\left(\left|\mathbf{u}_{n}\right|^{2}+\left|\mathbf{h}_{n}\right|^{2}\right) d S \leq C_{\epsilon} \nu_{n}^{2}
\end{gathered}
$$

hold, where $C_{\epsilon}$ is independent of $n$ and $\sigma$.

Now, for $(n, i)$-regular value $t \in\left[\frac{5}{8} t_{i}, \frac{7}{8} t_{i}\right]$, consider the domain

$$
\Omega_{i \overline{h_{n}}}(t)=\widetilde{W_{i n}(t)} \cup\left(\overline{\widetilde{V_{i+1}}} \backslash \overline{\Omega_{\overline{h_{n}}}}\right) .
$$

By construction, $\partial \Omega_{i \overline{h_{n}}}(t)=\Gamma_{\overline{h_{n}}} \cup \widetilde{S_{i n}}(t)$. Also using Equation (3.12), we know

$$
\begin{aligned}
\Delta \Phi_{n}= & \Delta p_{n}+\left|\nabla \mathbf{u}_{n}\right|^{2}+\left|\nabla \mathbf{h}_{n}\right|^{2}+\mathbf{u}_{n} \cdot \Delta \mathbf{u}+\mathbf{h}_{n} \cdot \Delta \mathbf{h}_{n} \\
= & -\operatorname{div}\left(\left(\mathbf{u}_{n} \cdot \nabla\right) \mathbf{u}_{n}\right)+\operatorname{div}\left(\left(\mathbf{h}_{n} \cdot \nabla\right) \mathbf{h}_{n}\right)+\left|\nabla \mathbf{u}_{n}\right|^{2}+\left|\nabla \mathbf{h}_{n}\right|^{2} \\
& -\frac{1}{\nu_{n}}\left(\left(\nabla \times \mathbf{f}_{n}\right) \cdot \mathbf{u}_{n}+\left(\nabla \times \mathbf{g}_{n}\right) \cdot \mathbf{h}_{n}\right) \\
& +\frac{1}{\nu_{n}}\left(\left(\mathbf{u}_{n} \cdot \nabla\right) \frac{\left|\mathbf{u}_{n}\right|^{2}}{2}+\mathbf{u}_{n} \cdot \nabla p_{n}-\mathbf{u}_{n} \cdot\left(\left(\mathbf{h}_{n} \cdot \nabla\right) \mathbf{h}_{n}\right)\right) \\
& +\frac{1}{\nu_{n}}\left(\left(\mathbf{u}_{n} \cdot \nabla\right) \frac{\left|\mathbf{h}_{n}\right|^{2}}{2}-\mathbf{h}_{n} \cdot\left(\left(\mathbf{h}_{n} \cdot \nabla\right) \mathbf{u}_{n}\right)\right) \\
=- & \sum_{i, j=1}^{3} \partial_{i} u_{n j} \partial_{j} u_{n i}+\left|\nabla \mathbf{u}_{n}\right|^{2}+\left|\nabla \mathbf{h}_{n}\right|^{2}+\sum_{i, j=1}^{3} \partial_{i} h_{n j} \partial_{j} h_{n i}+\frac{1}{\nu_{n}}\left(\mathbf{u}_{n} \cdot \nabla\right) \Phi_{n} \\
& -\frac{1}{\nu_{n}}\left(\mathbf{h}_{n} \cdot \nabla\right)\left(\mathbf{u}_{n} \cdot \mathbf{h}_{n}\right)-\frac{1}{\nu_{n}}\left(\left(\nabla \times \mathbf{f}_{n}\right) \cdot \mathbf{u}_{n}+\left(\nabla \times \mathbf{g}_{n}\right) \cdot \mathbf{h}_{n}\right) \\
= & \frac{1}{\nu_{n}} \operatorname{div}\left(\Phi_{n} \mathbf{u}_{n}\right)+\frac{1}{2}\left|\nabla \mathbf{u}_{n}-\left(\nabla \mathbf{u}_{n}\right)^{T}\right|^{2}+\frac{1}{2}\left|\nabla \mathbf{h}_{n}+\left(\nabla \mathbf{h}_{n}\right)^{T}\right|^{2} \\
& -\frac{1}{\nu_{n}}\left(\left(\nabla \times \mathbf{f}_{n}\right) \cdot \mathbf{u}_{n}+\left(\nabla \times \mathbf{g}_{n}\right) \cdot \mathbf{h}_{n}\right),
\end{aligned}
$$

where we have used the special structure of $\mathbf{u}_{n}$ and $\mathbf{h}_{n}$, so that $\left(\mathbf{h}_{n} \cdot \nabla\right)\left(\mathbf{u}_{n} \cdot \mathbf{h}_{n}\right) \equiv 0$. Integrating Equation (3.54) over the domain $\Omega_{i \overline{h_{n}}}(t)$, we obtain

$$
\begin{aligned}
& \int_{\widetilde{S_{i n}}} \nabla \Phi_{n} \cdot \mathbf{n} d s+\int_{\Gamma_{\overline{h_{n}}}} \nabla \Phi_{n} \cdot \mathbf{n} d s \\
= & \int_{\Omega_{i \overline{h_{n}}}(t)} \frac{1}{2}\left|\nabla \mathbf{u}_{n}-\left(\nabla \mathbf{u}_{n}\right)^{T}\right|^{2}+\frac{1}{2}\left|\nabla \mathbf{h}_{n}+\left(\nabla \mathbf{h}_{n}\right)^{T}\right|^{2} d x \\
& -\frac{1}{\nu_{n}} \int_{\Omega_{i \overline{h_{n}}}(t)}\left(\left(\nabla \times \mathbf{f}_{n}\right) \cdot \mathbf{u}_{n}+\left(\nabla \times \mathbf{g}_{n}\right) \cdot \mathbf{h}_{n}\right) d x+\frac{1}{\nu_{n}} \int_{\widetilde{S_{i n}}} \Phi_{n} \mathbf{u}_{n} \cdot \mathbf{n} d s+\frac{1}{\nu_{n}} \int_{\Gamma_{\overline{h_{n}}}} \Phi_{n} \mathbf{u}_{n} \cdot \mathbf{n} d s
\end{aligned}
$$




$$
\begin{aligned}
= & \int_{\Omega_{i \overline{h_{n}}}(t)} \frac{1}{2}\left|\nabla \mathbf{u}_{n}-\left(\nabla \mathbf{u}_{n}\right)^{T}\right|^{2}+\frac{1}{2}\left|\nabla \mathbf{h}_{n}+\left(\nabla \mathbf{h}_{n}\right)^{T}\right|^{2} d x \\
& -\frac{1}{\nu_{n}} \int_{\Omega_{\bar{i}_{\bar{n}}}(t)}\left(\left(\nabla \times \mathbf{f}_{n}\right) \cdot \mathbf{u}_{n}+\left(\nabla \times \mathbf{g}_{n}\right) \cdot \mathbf{h}_{n}\right) d x+\frac{1}{\nu_{n}} \int_{\Gamma_{\overline{h_{n}}}} \Phi_{n} \mathbf{u}_{n} \cdot \mathbf{n} d s-t \lambda_{n} \overline{\mathcal{F}},
\end{aligned}
$$

where $\overline{\mathcal{F}}=\left(\mathcal{F}_{M^{\prime}}+\cdots+\mathcal{F}_{M}\right)$. In view of Equation (3.52), we can estimate

$$
\begin{aligned}
\int_{\widetilde{S_{i n}}}\left|\nabla \Phi_{n}\right| d s \leq & t \mathcal{F}+\epsilon+\frac{1}{\nu_{n}} \int_{\Omega_{i \overline{h_{n}}}(t)}\left(\left(\nabla \times \mathbf{f}_{n}\right) \cdot \mathbf{u}_{n}+\left(\nabla \times \mathbf{g}_{n}\right) \cdot \mathbf{h}_{n}\right) d x \\
& -\int_{\Omega_{i \overline{h_{n}}}(t)}\left(\frac{1}{2}\left|\nabla \mathbf{u}_{n}-\left(\nabla \mathbf{u}_{n}\right)^{T}\right|^{2}+\frac{1}{2}\left|\nabla \mathbf{h}_{n}+\left(\nabla \mathbf{h}_{n}\right)^{T}\right|^{2}\right) d x \\
& +\frac{1}{\nu_{n}}\left(\int_{\Gamma_{\overline{h_{n}}}} \Phi_{n}^{2} d s\right)^{\frac{1}{2}}\left(\int_{\Gamma_{\overline{h_{n}}}}\left|\mathbf{u}_{n}\right|^{2} d s\right)^{\frac{1}{2}}
\end{aligned}
$$

with $\mathcal{F}=|\overline{\mathcal{F}}|$. By definition, $\frac{1}{\nu_{n}}\left\|\nabla \times \mathbf{f}_{n}\right\|_{L^{2}(\Omega)}=\lambda_{n} \nu_{n}\|\nabla \times \mathbf{f}\|_{L^{2}(\Omega)} \rightarrow 0$ as $n \rightarrow \infty$. Therefore,

$$
\left|\frac{1}{\nu_{n}} \int_{\Omega_{i \overline{h_{n}}}(t)}\left(\left(\nabla \times \mathbf{f}_{n}\right) \cdot \mathbf{u}_{n}+\left(\nabla \times \mathbf{g}_{n}\right) \cdot \mathbf{h}_{n}\right) d x\right| \leq \epsilon
$$

for sufficiently large $n$. Using inequalities (3.51) and (3.53) in Equation (3.55), we obtain

$$
\begin{aligned}
\int_{\widetilde{S_{i n}}}\left|\nabla \Phi_{n}\right| d s & \leq t \mathcal{F}+2 \epsilon+\sigma \sqrt{\overline{C_{\epsilon}}}-\int_{\Omega_{i \overline{h_{n}}}(t)} \frac{1}{2}\left|\nabla \mathbf{u}_{n}-\left(\nabla \mathbf{u}_{n}\right)^{T}\right|^{2}+\frac{1}{2}\left|\nabla \mathbf{h}_{n}+\left(\nabla \mathbf{h}_{n}\right)^{T}\right|^{2} d x \\
& \leq t \mathcal{F}+2 \epsilon+\sigma \sqrt{\overline{C_{\epsilon}}}-\int_{\widetilde{V_{i+1}} \backslash \Omega_{\delta_{i}}} \frac{1}{2}\left|\nabla \mathbf{u}_{n}-\left(\nabla \mathbf{u}_{n}\right)^{T}\right|^{2}+\frac{1}{2}\left|\nabla \mathbf{h}_{n}+\left(\nabla \mathbf{h}_{n}\right)^{T}\right|^{2} d x,
\end{aligned}
$$

where $C_{\epsilon}$ is independent of $n$ and $\sigma$. Choosing $\epsilon=\frac{1}{6} \epsilon_{i}, \sigma=\frac{\epsilon_{i}}{3 \sqrt{C_{\epsilon}}}$, and a sufficiently large $n$, from Equation (3.48) we obtain $2 \epsilon+\sigma \sqrt{C_{\epsilon}}-\int_{\widetilde{V_{i+1} \backslash} \backslash \Omega_{\delta_{i}}} \frac{1}{2}\left|\nabla \mathbf{u}_{n}-\left(\nabla \mathbf{u}_{n}\right)^{T}\right|^{2}+\frac{1}{2} \mid \nabla \mathbf{h}_{n}+$ $\left.\left(\nabla \mathbf{h}_{n}\right)^{T}\right|^{2} d x \leq 0$. We have finished the proof.

Now we can derive a contradiction by using the Co-area formula.

LEMma 3.11. Assume that $\Omega \subset \mathbb{R}^{3}$ is a bounded domain of type (1.1) with $C^{2}$ smooth boundary $\partial \Omega,(\nabla \times \mathbf{f}, \nabla \times \mathbf{g}) \in W_{A S}^{1,2}(\Omega) \times W_{A S_{O S}}^{1,2}(\Omega)$, and $(\mathbf{a}, \mathbf{b}) \in W_{A S}^{3 / 2,2}(\partial \Omega) \times$ $W_{A S O S}^{3 / 2,2}(\partial \Omega)$ satisfies conditions (1.3)-(1.4). Then assumptions (MHD-AX) and Equation (3.35) lead to a contradiction.

The proof of Lemma 3.11 can be obtained by slightly modifying the proof of Lemma 3.9 of [16], i.e., replacing Hausdorff measure $\mathcal{H}^{1}$ by $\mathcal{H}^{2}$ and the curves $S_{i n}(t)$ by the surfaces $\widetilde{S_{i n}}(t)$ in the corresponding integrals, and the details are omitted. Therefore, we have excluded the second case.

3.3.3. The case: $\sup _{x \in \Omega} \Phi(x)>\max _{j=0, \ldots, N} p_{j}$. Assume that Equation (3.32) is satisfied, and set $\sigma=\max _{j=0, \ldots, N} p_{j}$. Then we can find a compact connected set $F \subset \mathcal{D} \backslash A_{\mathrm{w}}$ such that $\operatorname{diam}(F)>0,\left.\psi\right|_{F}=$ const, and $\Phi(F)>\sigma$. We may assume that $\sigma<0$ and $\Phi(F)=0$. We still need to separate $F$ from $\partial \mathcal{D}$ by regular cycles and take a number $r_{0}>0$ such that $F \subset \mathcal{D}_{r_{0}}$, the open set $\mathcal{D}_{\epsilon}=\{(r, z) \in \mathcal{D}: r>\epsilon\}$ is connected for every $\epsilon \leq r_{0}$ and conditions (3.38) are satisfied. Then, for $\epsilon \in\left(0, r_{0}\right]$, we can consider the 
behavior of $\Phi$ on the Kronrod trees $T_{\psi, \epsilon}$ corresponding to the restrictions $\left.\psi\right|_{\overline{\mathcal{D}_{\epsilon}}}$. Denote by $F^{\epsilon}$ the element of $T_{\psi, \epsilon}$ containing $F$. Using the same procedure as before, we can find $r_{*} \in\left(0, r_{0}\right]$ and $C_{j} \in\left[B_{j}^{r_{*}}, F^{r_{*}}\right], j=0, \ldots, N$, such that $\Phi\left(C_{j}\right)<0$ and $C \cap L_{r_{*}}=\emptyset$ for all $C \in\left[C_{j}, F^{r *}\right]$.

Set $T_{\psi}=T_{\psi, r_{*}}, F^{*}=F^{r_{*}}$, and $B_{j}=B_{j}^{r_{*}}$, i.e., $B_{j} \in T_{\psi}$ and $B_{j} \supset \breve{\Gamma}_{j} \cap \overline{\mathcal{D}_{r_{*}}}$. As above, we can change $C_{j}$ so that

$$
\begin{gathered}
\forall j=0, \ldots, N \quad C_{j} \in\left[B_{j}, F^{*}\right], \quad \Phi\left(C_{j}\right)<0, \\
C \cap \partial \mathcal{D}_{r_{*}}=\emptyset \quad \forall C \in\left[C_{j}, F^{*}\right], \quad \text { and } \quad C_{0}=\cdots=C_{M^{\prime}}
\end{gathered}
$$

Similarly, we should construct an appropriate integration domain by using the level sets of $\Phi$ and $\Phi_{n}$. Take positive numbers $t_{i}=2^{-i} t_{0}$, regular cycles $A_{i}^{j} \in\left[C_{j}, F^{*}\right]$ with $\Phi\left(A_{i}^{j}\right)=-t_{i}$, and the set $S_{i n}(t)$ with $\left.\Phi_{n}\right|_{S_{i n}(t)} \equiv-t$ separating $A_{i}^{M^{\prime}} \cup \cdots \cup A_{i}^{N}$ from $A_{i+1}^{M^{\prime}} \cup \cdots \cup A_{i+1}^{N}$, etc. Argued in Lemma 3.10 and Lemma 3.11, we can derive a similar contradiction as before. Therefore, we have finished the proof of Theorem 1.1.

Acknowledgement. Weng's research was supported by the Basic Science Research Program through the National Research Foundation of Korea (NRF) funded by the Ministry of Education, Science and Technology (No. 2015049582). The author would like to thank Prof. Dongho Chae and Prof. Zhouping Xin for their interest in this work and for their constant encouragement and support. The author wants to thank the referees for their careful reading and important suggestions and improvements.

\section{REFERENCES}

[1] C.J. Amick, Existence of solutions to the nonhomogeneous steady Navier-Stokes equations, Indiana Univ. Math. J., 33:817-830, 1984.

[2] W. Borchers and K. Pileckas, Note on the flux problem for stationary Navier-Stokes equations in domains with multiply connected boundary, Acta Appl. Math., 37:21-30, 1994.

[3] J. Bourgain, M. Korobkov, and J. Kristensen, On the Morse-Sard property and level sets of Sobolev and BV functions, Rev. Mat. Iberoam., 29(1):1-23, 2013.

[4] D. Chae and S. Weng, Liouville type theorems for the steady axially symmetric Navier-Stokes and magnetohydrodynamic equations, arXiv:1512.03491. Discrete Contin. Dyn. Syst. Ser. A, accepted.

[5] L.C. Evans and R.F. Gariepy, Measure Theory and Fine Properties of Functions, CRC, Boca Raton, FL, 1992.

[6] R. Finn, On the steady-state solutions of the Navier-Stokes equations. III, Acta Math., 105:197$244,1961$.

[7] H. Fujita, On the existence and regularity of the steady-state solutions of the Navier-Stokes theorem, J. Fac. Sci. Univ. Tokyo Sect. I, 9:59-102, 1961.

[8] H. Fujita, On stationary solutions to Navier-Stokes equation in symmetric plane domain under general outflow condition, Pitman Research Notes in Mathematics. Proceedings of International Conference on Navier-Stokes Equations. Theory and Numerical Methods, Varenna, Italy, 388:16-30, 1997.

[9] G.P. Galdi, On the existence of steady motions of a viscous flow with non-homogeneous conditions, Le Mathematiche 66:503-524, 1991.

[10] G.P. Galdi, An Introduction to the Mathematical Theory of the Navier-Stokes Equations. In: Steady State problems, Springer Monographs in Mathematics, Second Edition, 2011.

[11] E. Hopf, Ein allgemeiner Endlichkeitssats der Hydrodynamik, Math. Ann., 117:764-775, 1941.

[12] L.V. Kapitanskii, and K. Pileckas, On spaces of solenoidal vector fields and boundary value problems for the Navier-Stokes equations in domains with noncompact boundaries, Trudy Mat. Inst. Steklov, 159:5-36, 1983. English Transl.: Proc. Math. Inst. Steklov, 159:3-34, 1984.

[13] M. Korobkov, K. Pileckas, and R. Russo, On the flux problem in the theory of steady Navier-Stokes equations with nonhomogeneous boundary conditions, Arch. Ration. Mech. Anal., 207:185-213, 2013. 
[14] M. Korobkov, K. Pileckas, and R. Russo, The existence of a solution with finite Dirichlet integral for the steady Navier-Stokes equations in a plane exterior symmetric domain, J. Math. Pure. Appl., 101:257-274, 2014.

[15] M. Korobkov, K. Pileckas, and R. Russo, An existence theorem for steady Navier-Stokes equations in the axially symmetric case, Ann. Scuola Norm. Sup. Pisa Cl. Sci., XIV(1):233-262, 2015.

[16] M. Korobkov, K. Pileckas, and R. Russo, Solution of Leray's problem for the stationary NavierStokes equations in plane and axially symmetric spatial domains, Annals of Mathematics, 181:769-807, 2015.

[17] M. Korobkov, K. Pileckas, and R. Russo, The existence theorem for the steady Navier-Stokes problem in exterior axially symmetric 3D domains, arXiv: 1403.6921v1.

[18] H. Kozono and T. Yanagisawa, Leray's problem on the stationary Navier-Stokes equations with inhomogeneous boundary data, Math. Z., 262:27-39, 2009.

[19] A.S. Kronrod, On functions of two variables, Uspekhi Math. Nauk, 5:24-134, 1950.

[20] O.A. Ladyzhenskaya, The Mathematical Theory of Viscous Incompressible Fulid, Gordon and Breach, 1969.

[21] J. Leray, Étude de diverses équations intégrales non linéaire et de quelques problèmes que pose l'hydrodynamique, J. Math. Pures Appl., 12:1-82, 1933.

[22] H. Morimoto and H. Fujita, A remark on the existence of steady Navier-Stokes flows in a certain two-dimensional infinite channel, Tokyo J. Math., 25(2):307-321, 2002.

[23] H. Morimoto, Stationary Navier-Stokes equations with non-vanishing outflow condition, Hokkaido Math. J., 24(3):641-648, 1995.

[24] K. Pileckas and R. Russo, On the existence of vanishing at infinity symmetric solutions to the plane stationary exterior Navier-Stokes problem, Math. Ann., 352:643-658, 2012.

[25] A. Russo and G. Starita, On the existence of steady-state solutions to the Navier-Stokes system for large fluxes, Ann. Sc. Norm. Super. Pisa Cl. Sci., 7:171-180, 2008. 